\title{
Quantum gate teleportation between separated qubits in a trapped-ion processor
}

\author{
Yong Wan, ${ }^{1,2 * \dagger}$ Daniel Kienzler, ${ }^{1,2 \dagger \ddagger}$ Stephen D. Erickson, ${ }^{1,2}$ Karl H. Mayer, ${ }^{1,2}$ \\ Ting Rei Tan, ${ }^{1,2 \ddagger}$ Jenny J. Wu, ${ }^{1,2}$ Hilma M. Vasconcelos, ${ }^{1,2,4}$ Scott Glancy, ${ }^{1}$ \\ Emanuel Knill, ${ }^{1}$ David J. Wineland, ${ }^{1,2,3}$ Andrew C. Wilson, ${ }^{1}$ Dietrich \\ Leibfried $^{1}$ \\ ${ }^{1}$ National Institute of Standards and Technology, 325 Broadway, Boulder, CO \\ 80305, USA \\ ${ }^{2}$ Department of Physics, University of Colorado, Boulder, CO 80309, USA \\ ${ }^{3}$ Department of Physics, University of Oregon, Eugene, OR 97403, USA \\ ${ }^{4}$ Departamento de Engenharia de Teleinformática, Universidade Federal do \\ Ceará, Fortaleza, Brazil \\ *To whom correspondence should be addressed. E-mail: yong.wan@ @nist.gov. \\ ${ }^{\dagger}$ These authors contributed equally to this work. ${ }^{\ddagger}$ Current address: ETH \\ Zurich, Otto-Stern-Weg 1, HPF E10, 8093 Zurich, Switzerland (D.K.); \\ Department of Physics, National University of Singapore, 2 Science Drive 3, \\ 117551 Singapore (T.R.T); Centre for Quantum Technologies, 3 Science Drive \\ 2, 117543 Singapore (T.R.T.).
}

Large-scale quantum computers will require quantum gate operations between widely separated qubits. A method for implementing such operations, known as quantum gate teleportation (QGT), requires only local operations, classical communication, and shared entanglement. We demonstrate QGT in a scalable architecture by deterministically teleporting a controlled-NOT (CNOT) gate between two qubits in spatially separated locations in an ion trap. The 
entanglement fidelity of our teleported CNOT is in the interval $[0.845,0.872]$ at the $95 \%$ confidence level. The implementation combines ion shuttling with individually-addressed single-qubit rotations and detections, same- and mixedspecies two-qubit gates, and real-time conditional operations, thereby demonstrating essential tools for scaling trapped-ion quantum computers combined in a single device.

Quantum computers have the potential to solve problems that are intractable using conventional computers. However, many quantum bits (qubits) are required to outperform conventional computing capabilities, and scaling quantum computers to be practically useful is difficult (1). As the system size increases, the average distance between qubits grows, making it harder to connect arbitrary qubits. Quantum gate teleportation (QGT) is a uniquely quantum solution that enables logical gates between spatially separated qubits, where shared entanglement eliminates the need for a direct quantum coherent interaction $(2,3)$.

There are several proposals for scaling up to larger numbers of qubits in trapped-ion systems. These include the "quantum charge-coupled device" (QCCD) architecture, which incorporates a large array of segmented electrodes to create trapping zones specialized for roles such as loading ions, processing, and memory storage $(4,5)$. Qubits can interact by being physically moved to the same zone. A variant of this approach couples different zones by creating entanglement via a photonic network (6). Both approaches will benefit from a way to perform gate operations between separated qubits via QGT, which mitigates latency from transmitting quantum information between zones, provided that the required entangled ancilla pairs are prepared and distributed ahead of time during unrelated processor functions. This entanglement can be produced using various methods, including unitary gates, dissipative schemes (7), and photonic links (8).

Progress towards distributed quantum computation has been made with quantum state tele- 
portation (9), where an arbitrary state is transferred between remote parties $(2,10)$. Using state teleportation, a two-qubit gate between two parties, Alice and Bob, can be implemented by teleporting Alice's input state to Bob, applying local two-qubit gates at Bob's location, and teleporting Alice's half of the output back to her. This process consumes a minimum of two shared entangled pairs.

For a controlled-NOT (CNOT) gate, the task can be achieved more efficiently using the protocol depicted in Fig. 1A (3), without the need to physically bring the qubits together or teleport the states back and forth. This protocol achieves the minimum possible overhead, requiring only a single entangled pair shared between two locations, local operations, and classical communication. The protocol implementing a teleported CNOT between qubits $\mathrm{B}_{1}$ and $\mathrm{B}_{2}$ works as follows. The initial entanglement between qubits $M_{1}$ and $M_{2}$ is transferred to $B_{1}$ and $M_{2}$ through the first local CNOT, $\mathrm{M}_{1}$ detection, and conditional operation on $\mathrm{M}_{2}$. With the information about $\mathrm{B}_{1}$ 's state now shared with $\mathrm{M}_{2}, \mathrm{~B}_{1}$ is the effective control of the second local CNOT that acts on $B_{2}$. The remaining operations serve to disentangle $M_{2}$ from $B_{1}$ and $B_{2}$, resulting in an effective $C N O T$ between $\mathrm{B}_{1}$ and $\mathrm{B}_{2}$.

This type of teleported gate has been demonstrated probabilistically with photonic systems, where the required conditional operations were implemented with passive optical elements and post-selection $(11,12)$. More recently, a deterministic CNOT was teleported between two superconducting cavity qubits using an entangled pair of transmons (13).

Here, we demonstrate a deterministic teleported CNOT between two ${ }^{9} \mathrm{Be}^{+}$ions using a shared entangled pair of ${ }^{25} \mathrm{Mg}^{+}$ions. It combines key elements for scalable quantum computation with trapped ions, including separation and transport of mixed-species ion crystals, local same- and mixed-species two-qubit gates (14), individually-addressed single-qubit rotations and detection, and conditional operations based on measurement results. We use quantum process tomography (QPT) to characterize the teleported CNOT. We simplify the demonstra- 
tion by using only one laser interaction zone (LIZ) and transporting the separated qubits to this location, but the key elements of the protocol are retained.

Our experiment uses two ${ }^{9} \mathrm{Be}^{+}$ions $\left(\mathrm{B}_{1}, \mathrm{~B}_{2}\right)$ and two ${ }^{25} \mathrm{Mg}^{+}$ions $\left(\mathrm{M}_{1}, \mathrm{M}_{2}\right)$ trapped in a segmented linear Paul trap. The qubits are encoded in the $\left|F=1, m_{F}=1\right\rangle_{\mathrm{B}} \equiv|\uparrow\rangle_{\mathrm{B}}$ and $|2,0\rangle_{\mathrm{B}} \equiv|\downarrow\rangle_{\mathrm{B}}$ hyperfine states of ${ }^{9} \mathrm{Be}^{+}$and the $|2,0\rangle_{\mathrm{M}} \equiv|\uparrow\rangle_{\mathrm{M}}$ and $|3,1\rangle_{\mathrm{M}} \equiv|\downarrow\rangle_{\mathrm{M}}$ states of ${ }^{25} \mathrm{Mg}^{+}$. We use the symbol $\mathrm{B}(\mathrm{M})$ for ${ }^{9} \mathrm{Be}^{+}\left({ }^{25} \mathrm{Mg}^{+}\right)$ions, and label respective states with subscript B (M). To begin each experiment, a four-ion chain is initialized in the order $\mathrm{B}_{1}-\mathrm{M}_{1}-$ $\mathrm{M}_{2}-\mathrm{B}_{2}$ in a potential well where all laser beams pass through the trap, a region we refer to as the LIZ (Fig. 2). Qubit-state measurements of B (M) ions are realized by state-dependent fluorescence detection with $313 \mathrm{~nm}(280 \mathrm{~nm})$ resonant light after transferring the population from the computational basis to the measurement basis $|\uparrow\rangle_{\mathrm{B}} \rightarrow|2,2\rangle_{\mathrm{B}} \equiv \mid$ Bright $\rangle_{\mathrm{B}}$ and $|\downarrow\rangle_{\mathrm{B}} \rightarrow$ $|1,-1\rangle_{\mathrm{B}} \equiv|\operatorname{Dark}\rangle_{\mathrm{B}}\left(|\downarrow\rangle_{\mathrm{M}} \rightarrow|3,3\rangle_{\mathrm{M}} \equiv \mid \text { Bright }\right\rangle_{\mathrm{M}}$ and $|\uparrow\rangle_{\mathrm{M}} \rightarrow|2,-2\rangle_{\mathrm{M}} \equiv \mid$ Dark $\left.\rangle_{\mathrm{M}}\right)(15)$. Segmented trap electrodes enable the use of time-varying potentials to split the ion crystal into selected subsets and to transport them to and from the $\operatorname{LIZ}(16,17)$. Spatial separation enables individual addressing of ions of the same species, while ions of different species are distinguished by their well-separated resonant wavelengths.

Stimulated Raman transitions are used for all coherent qubit operations. A pair of copropagating laser beams for each species drives single-qubit rotations $\hat{R}(\theta, \phi)$, and rotation around the $z$-axis $\hat{R}_{\mathrm{Z}}(\alpha)$ is implemented by phase-shifting all subsequent single-qubit rotations for that qubit (15). A pair of perpendicular laser beams for each species drives twoqubit Mølmer-Sørensen (MS) entangling gates (18). Both pairs of Raman beams are applied simultaneously to drive mixed-species MS gates (14). We construct $\mathrm{CNOT}_{\mathrm{C} \rightarrow \mathrm{T}}$ (C for control and $\mathrm{T}$ for target) and the Bell-state-generating gate $\hat{F}$ using single-qubit rotations and MS gates $(14,15,19)$.

The circuit diagram for our teleported CNOT is shown in Fig. 1B, and ion configurations 
during QGT are illustrated in Fig. 2. After ground-state cooling the four-ion chain, the algorithm begins with the $\mathrm{B}$ and $\mathrm{M}$ ions in $|\mathrm{Bright}\rangle_{\mathrm{B}}$ and $|\downarrow\rangle_{\mathrm{M}}$, respectively. $\hat{F}$ is applied to the two $\mathrm{M}$ ions to generate the Bell state $\left|\Phi^{+}\right\rangle_{\mathrm{M}}=\frac{1}{\sqrt{2}}\left(|\uparrow \uparrow\rangle_{\mathrm{M}}+|\downarrow \downarrow\rangle_{\mathrm{M}}\right)$ (Fig. 2A). Afterwards, the chain is split into two B-and-M pairs in separated regions of a double-well potential (Fig. 2B) which is translated to bring $B_{1}-M_{1}$ into the LIZ. There we ground-state cool $B_{1}-M_{1}$ by addressing $B_{1}$, prepare $B_{1}$ to its input state, and apply $\mathrm{CNOT}_{\mathrm{B}_{1} \rightarrow \mathrm{M}_{1}}$. Then, $\mathrm{M}_{1}$ is detected (Fig. 2C). Its qubit state is determined by comparing the number of detected photons to a preset threshold. The double-well potential is then translated to move $\mathrm{M}_{2}-\mathrm{B}_{2}$ into the LIZ, where the pair is ground-state cooled by addressing $\mathrm{B}_{2}$. Qubit $\mathrm{B}_{2}$ is then prepared in its input state, and the conditional operation $\hat{R}(\pi, 0)$ is applied on $\mathrm{M}_{2}$ if $\mathrm{M}_{1}$ was measured to be in $|\downarrow\rangle_{\mathrm{M}}$. Next we apply $\mathrm{CNOT}_{\mathrm{M}_{2} \rightarrow \mathrm{B}_{2}}$, followed by a rotation $\hat{R}\left(\frac{\pi}{2},-\frac{\pi}{2}\right)$ and detection of $\mathrm{M}_{2}$. A rotation selecting the measurement axis for state tomography is applied to $\mathrm{B}_{2}$, which is then mapped out to the measurement basis, but not yet detected (Fig. 2D). This mapping reduces the depumping of $\mathrm{B}_{2}$ from stray scattered light when detecting $\mathrm{B}_{1}$ later in the process. The double-well potential is translated back to bring $\mathrm{B}_{1}-\mathrm{M}_{1}$ into the LIZ, where we apply the conditional operation $\hat{R}_{\mathrm{Z}}(\pi)$ if $\mathrm{M}_{2}$ was measured to be in $|\downarrow\rangle_{\mathrm{M}}$, followed by a single-qubit rotation selecting the measurement axis and a measurement of $B_{1}$ (Fig. 2E). Subsequently, $M_{2}-B_{2}$ are shuttled back into the LIZ where $\mathrm{B}_{2}$ is detected (Fig. 2F). At the end of this sequence, the four ions are recombined into a single well to prepare for the next repetition of the experiment.

We used QPT (20) to characterize our teleported CNOT between the two B ions. 144 different combinations of input states and measurement axes were implemented in random order and each for approximately 300 consecutive experiment executions. Two complete sets of tomography data were acquired. We developed a protocol for data analysis on Data Set 1 while remaining blind to Data Set 2, and then applied this protocol to Data Set 2. We summarize the analysis methods and results for Data Set 2 below. 
From the observed measurement outcomes, we determined the most likely quantum process by maximum likelihood (ML) estimation, and inferred a 95\% confidence interval of $[0.845$, 0.872] for the entanglement fidelity with respect to an ideal CNOT. The matrix representing the quantum process is shown in Fig. 3. For details of our analysis, see (15).

Ideally, the observed data should be consistent with the assumption of a single quantum process, but drifts in control parameters on time-scales much slower than a single QGT experiment can lead to imperfections. To detect departure from this assumption, we applied a likelihood ratio (LR) test $(21,22)$. An $\mathrm{LR}$ was computed from the experimental data and compared to the distribution of LRs obtained from synthetic datasets generated by parametric bootstrapping (23). The test indicated that our data was inconsistent with a single quantum process (15). Motivated by this finding, we discovered drifts in single-qubit-rotation angles that eluded our feedback mechanisms but can be addressed in future experiments (15). We verified through numerical simulation that realistic fluctuations of single-qubit-rotation angles are capable of causing an inconsistency comparable to that observed in our data. Although such drifts are not major sources of infidelity for this experiment (15), such consistency checks could be an important diagnostic that can supplement other benchmarking techniques and uncover overlooked sources of infidelity.

We list the dominant error sources and estimate their combined impact in Table 1. If all errors are mutually independent, the total error is $0.16(2)$. A more accurate description of the impact of individual errors using a depolarizing model predicts a process fidelity of $0.88(1)$, which is near the upper limit of the 95\% ML confidence interval, indicating that the major error sources are included in the error propagation model (15).

Ideally one would implement QGT using an ion species with a transition insensitive to magnetic field fluctuations to serve as both information-carrying qubits and entanglement-resource qubits, and a second dedicated species for cooling. This would mitigate decoherence and allow 
Table 1: Error sources for the teleported CNOT. The Bell-state fidelity with SPAM error contributions subtracted is used as an estimate of the mixed-species CNOT fidelity (15). $1 \sigma$ uncertainty for the respective error sources are shown in parentheses.

\begin{tabular}{ll}
\hline Source & Error $\left(10^{-2}\right)$ \\
\hline \hline SPAM on two B ions & $1.1(7)$ \\
SPAM on two M ions & $1.5(3)$ \\
$\mathrm{M}_{1}-\mathrm{M}_{2}$ Bell state & $4.0(9)$ \\
$\mathrm{B}_{1}-\mathrm{M}_{1}$ CNOT & $3.0(9)$ \\
$\mathrm{M}_{2}-\mathrm{B}_{2}$ CNOT & $3(1)$ \\
Coherence of M ions & $0.7(3)$ \\
Stray light from $\mathrm{M}_{1}$ detection on $\mathrm{M}_{2}$ & $1.1(4)$ \\
Stray light from $\mathrm{B}_{2}$ cooling on $\mathrm{B}_{1}$ & $1.2(3)$ \\
\hline Sum & $16(2)$ \\
Depolarizing model & $12(1)$ \\
\hline
\end{tabular}

QGT to be embedded in a larger quantum circuit (in our experiment any prior information encoded in $\mathrm{B}_{1}$ and $\mathrm{B}_{2}$ would be destroyed during cooling). Errors from stray light scattering could also be removed by using the coolant species for quantum logic readout (24). To be viable for fault-tolerant error correction, larger algorithms like QGT will also require constituent operations to be performed with higher fidelity in multiple locations. A larger QCCD array would then have many different interaction zones and integrated detection zones (25). The fact that our experimental duty cycle was dominated by shuttling and associated recooling (15) emphasizes the importance of cold diabatic transport $(26,27)$ and faster cooling techniques $(28)$.

Deterministic teleported CNOT gates can serve as a useful primitive for large-scale quantum computation. The integration of several operations, including mixed-species coherent control, ion transport, and entangling operations on selected subsets of qubits will be essential for building large-scale quantum computers based on ions in the QCCD architecture. Moreover, applying consistency checks to the experimental data facilitated the identification of error sources in the experimental setup, illustrating the importance of performing such checks in addition to 
tomography when characterizing quantum processes. Similar consistency checks could be done between disjunct processing nodes executing the same routine, exposing compromised nodes that behave differently from the rest.

\section{References and Notes}

1. J. Preskill, Quantum 2, 79 (2018).

2. D. Gottesman, I. L. Chuang, Nature 402, 390 (1999).

3. J. Eisert, K. Jacobs, P. Papadopoulos, M. B. Plenio, Physical Review A 62, 052317 (2000).

4. D. J. Wineland, et al., Journal of Research of the National Institute of Standards and Technology 103, 259 (1998).

5. D. Kielpinski, C. Monroe, D. J. Wineland, Nature 417, 709 (2002).

6. C. Monroe, et al., Physical Review A 89, 022317 (2014).

7. Y. Lin, et al., Nature 504, 415 (2013).

8. D. L. Moehring, et al., Nature 449, 68 (2007).

9. S. Pirandola, J. Eisert, C. Weedbrook, A. Furusawa, S. L. Braunstein, Nature Photonics 9 , $641(2015)$

10. C. H. Bennett, et al., Physical Review Letters 70, 1895 (1993).

11. Y.-F. Huang, X.-F. Ren, Y.-S. Zhang, L.-M. Duan, G.-C. Guo, Physical Review Letters 93, 240501 (2004).

12. W.-B. Gao, et al., Proceedings of the National Academy of Sciences 107, 20869 (2010). 
13. K. S. Chou, et al., Nature 561, 368 (2018).

14. T. R. Tan, et al., Nature 528, 380 (2015).

15. Materials and methods are available as supplementary materials at the Science website.

16. R. B. Blakestad, et al., Phys. Rev. Lett. 102, 153002 (2009).

17. R. B. Blakestad, et al., Physical Review A 84, 032314 (2011).

18. A. Sørensen, K. Mølmer, Phys. Rev. Lett. 82, 1971 (1999).

19. P. J. Lee, et al., Journal of Optics B: Quantum and Semiclassical Optics 7, S371 (2005).

20. I. L. Chuang, M. A. Nielsen, Journal of Modern Optics 44, 2455 (1997).

21. G. Casella, R. L. Berger, Statistical Inference (Cengage Learning, 2001), p. 374.

22. R. Blume-Kohout, et al., Nature Communications 8, 14485 (2017).

23. B. Efron, R. J. Tibshirani, An Introduction to the Bootstrap (CRC Press, 1994).

24. P. O. Schmidt, et al., Science 309, 749 (2005).

25. D. H. Slichter, et al., Optics Express 25, 8705 (2017).

26. R. Bowler, et al., Phys. Rev. Lett. 109, 080502 (2012).

27. A. Walther, et al., Phys. Rev. Lett. 109, 080501 (2012).

28. C. F. Roos, et al., Physical Review Letters 85, 5547 (2000).

29. Y. Wan, Data for "Quantum gate teleportation between separated qubits in a trappedion processor," Version 1, National Institute of Standards and Technology (2019); http://doi.org/10.18434/M32056. 
30. J. P. Gaebler, et al., Phys. Rev. Lett. 117, 060505 (2016).

31. C. Monroe, et al., Physical Review Letters 75, 4011 (1995).

32. Y. Wan, F. Gebert, F. Wolf, P. O. Schmidt, Physical Review A 91, 043425 (2015).

33. A. C. Keith, C. H. Baldwin, S. Glancy, E. Knill, Phys. Rev. A 98, 042318 (2018).

34. M.-D. Choi, Linear Algebra and its Applications 10, 285 (1975).

35. J. Fiurek, Physical Review A 64, 024102 (2001).

36. B. Schumacher, Physical Review A 54, 2614 (1996).

37. T. L. Scholten, R. Blume-Kohout, New Journal of Physics 20, 023050 (2018).

38. K. Rudinger, et al., arXiv:1810.05651 (2018).

39. C. A. Sackett, et al., Nature 404, 256 (2000).

40. C. J. Ballance, High-Fidelity Quantum Logic in $\mathrm{Ca}^{+}$, Thesis, University of Oxford (2014).

\section{Acknowledgments}

We thank P. Hou and D. Cole of the NIST Ion Storage Group for helpful comments on the manuscript. We thank D. T. C. Allcock, S. C. Burd (both of the NIST Ion Storage Group), the Oxford University, and the ETH Zürich ion trapping groups for their advice on stabilizing magnetic fields. Funding: This work was supported by the Office of the Director of National Intelligence (ODNI) Intelligence Advanced Research Projects Activity (IARPA), ONR, and the NIST Quantum Information Program. D.K. acknowledges support from the Swiss National Science Foundation under grant no. 165208. S.D.E. acknowledges support by the U.S. National Science Foundation under Grant No. DGE 1650115. Y.W., D.K., J.J.W., and H.M.V. 
are associates in the Professional Research Experience Program (PREP) operated jointly by NIST and University of Colorado Boulder. H.M.V. acknowledges support from the Schlumberger Foundation's Faculty for the Future program. Author contributions: Y.W. and D.K. performed the majority of experiments with assistance from S.D.E., T.R.T, and J.J.W.. D.J.W., A.C.W., and D.L. advised on the project. K.H.M., H.M.V., S.G., and E.K. developed statistical models and provided theorectical support. Y.W., D.K., and K.H.M. contributed to data analysis. Y.W., S.D.E., and K.H.M. wrote the manuscript. All authors revised and commented on the manuscript. Competing interests: The authors declare that there are no competing financial interests. Data and materials availability: Data from the main text and supplementary materials are available through NIST public data repository (29).

\section{List of Supplementary Materials}

Supplementary Text

Figure S1-S9

Table S1-S3

References (30-40) 
(A)
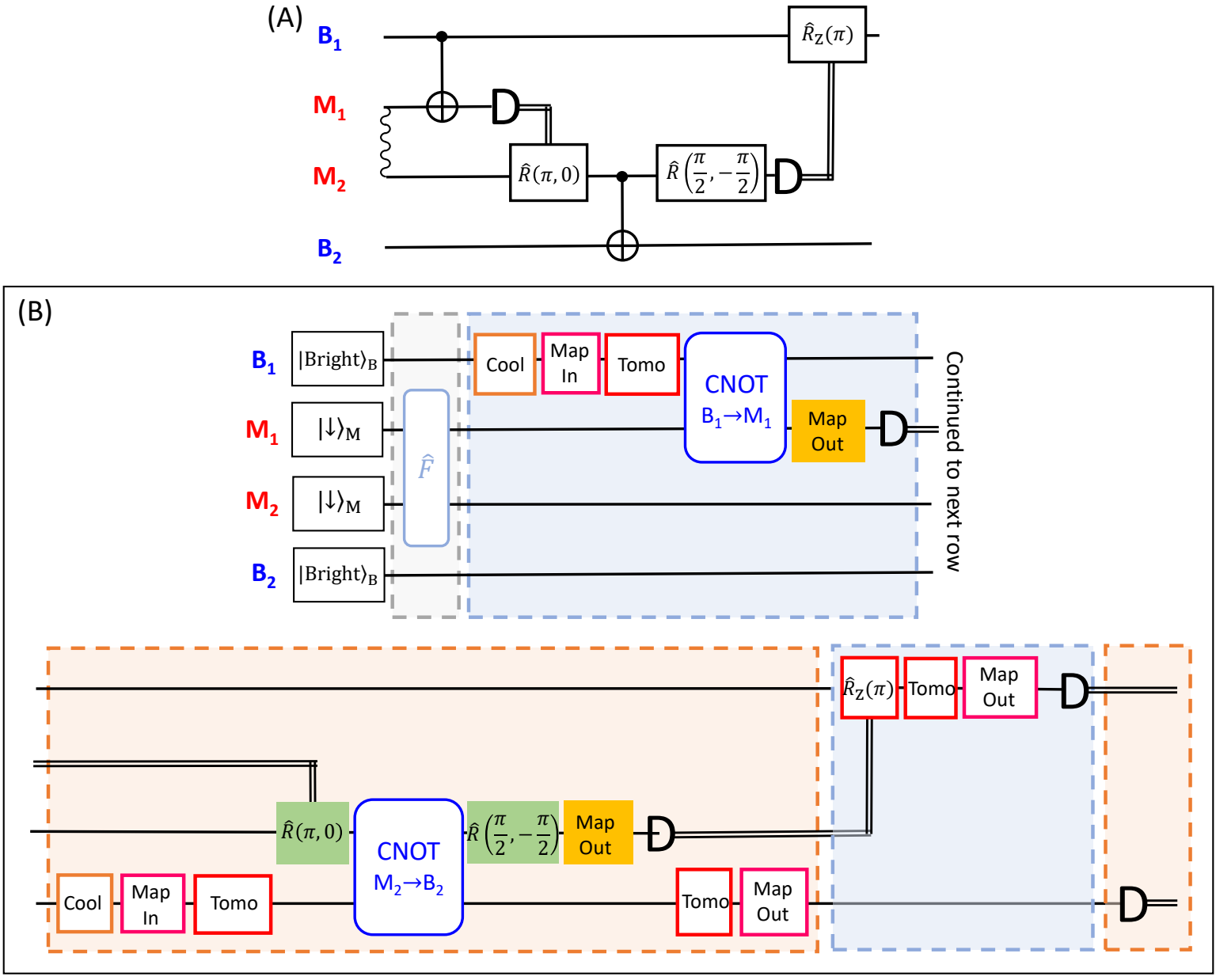

Figure 1: QGT circuit diagram. (A) Circuit diagram for a teleported CNOT between qubits $\mathrm{B}_{1}$ and $\mathrm{B}_{2}$ as proposed in (3). The wavy line represents entanglement, and double solid lines represent classical communication. (B) Experiment-specific circuit diagram for the teleported CNOT gate between $\mathrm{B}_{1}$ and $\mathrm{B}_{2}$. "Map In" indicates mapping from the measurement basis to the computational basis, while "Map Out" indicates the opposite process. "Tomo" refers to single-qubit rotations for QPT. 


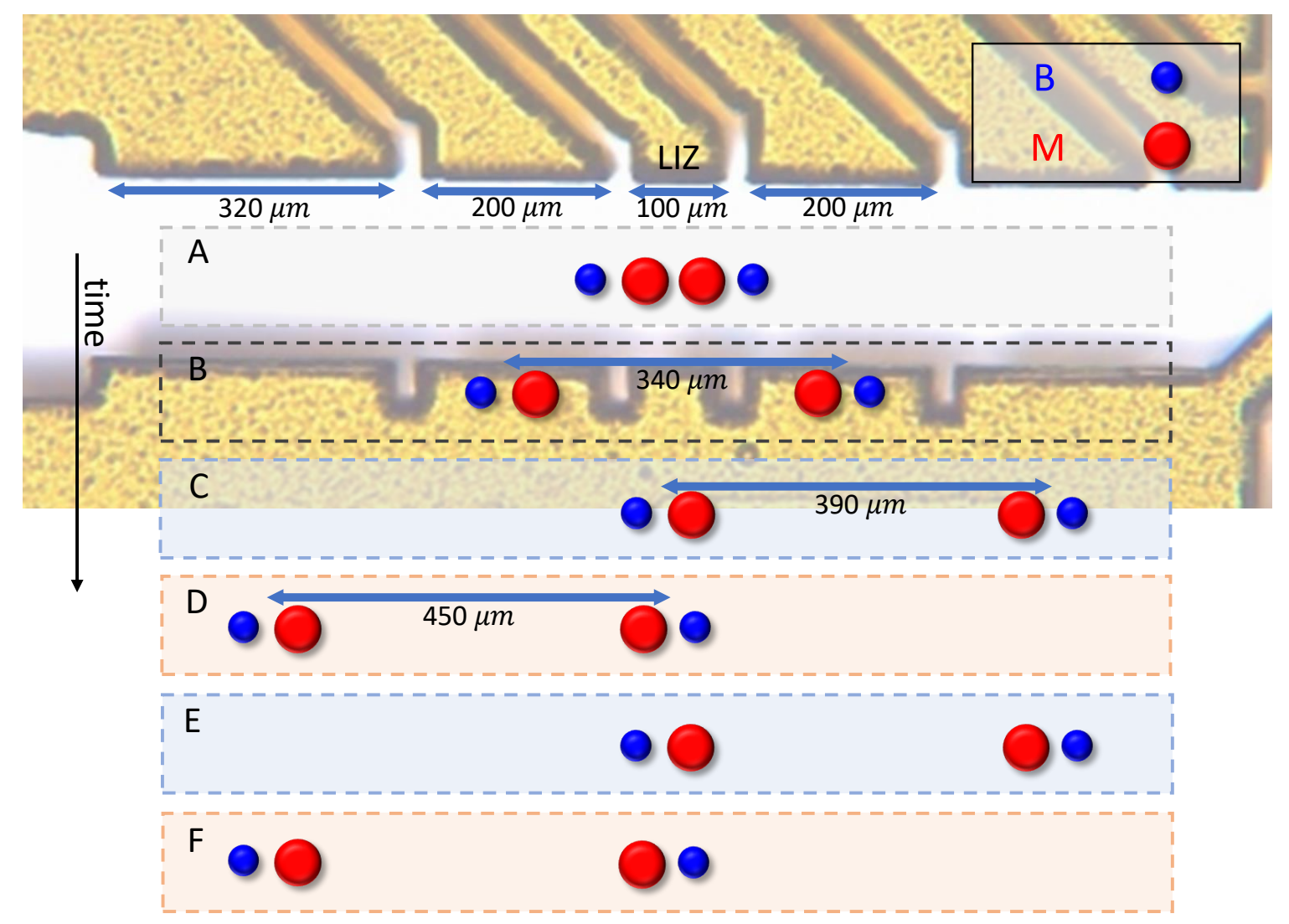

Figure 2: QGT shuttling sequence. Panels A-F show the shuttling sequence, overlaid on a photograph of a section of the trap electrode structure (ions and ion spacings are not to scale). After preparing the $\mathrm{M}$ ions in a Bell state to serve as the entanglement resource, the $\mathrm{B}_{1}-\mathrm{M}_{1}-$ $\mathrm{M}_{2}-\mathrm{B}_{2}$ chain is split into two pairs of B-and-M ions, which are translated into and out of LIZ to address and detect individual ions (blocks C, D, E and F). 

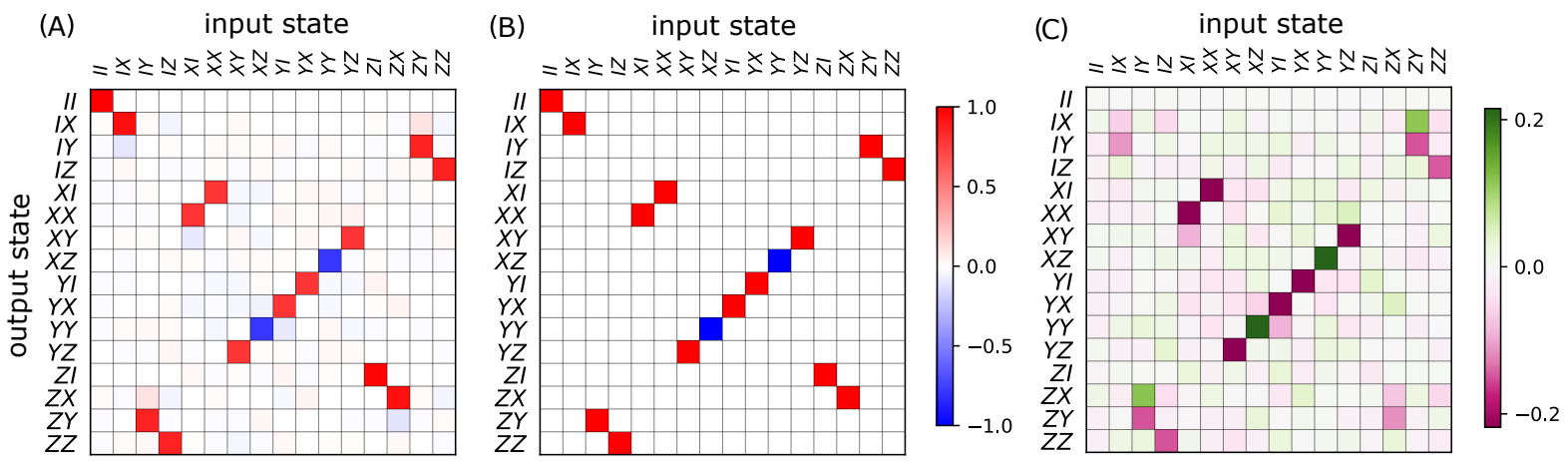

Figure 3: Pauli transfer matrix. Visualization of quantum processes in the Pauli transfer matrix representation for (A) the experimental data "Data Set 2", (B) the ideal CNOT process, and (C) the difference between the experimental and ideal process matrices (15). The Pauli transfer matrix of a process maps an arbitrary input density matrix, expressed as a linear combination of Pauli products, into the corresponding linear combination that describes the output density matrix. For our implementation, a 95\% confidence interval [0.845, 0.872] for the entanglement fidelity is determined with respect to an ideal CNOT. 


\section{Supplementary Text}

Trap and Qubit This experiment is performed in a multi-layer segmented linear Paul trap, described in detail in (17). We use ${ }^{9} \mathrm{Be}^{+}$and ${ }^{25} \mathrm{Mg}^{+}$as qubits at a magnetic field of $1.19 \times 10^{-2} \mathrm{~T}$. The detailed level structures for both qubits are depicted in Fig. S1. The qubits are encoded in the two levels of the hyperfine groundstate manifolds $|1,1\rangle_{\mathrm{B}} \equiv|\uparrow\rangle_{\mathrm{B}}$ and $|2,0\rangle_{\mathrm{B}} \equiv|\downarrow\rangle_{\mathrm{B}}$ for ${ }^{9} \mathrm{Be}^{+}$ions and $|2,0\rangle_{\mathrm{M}} \equiv|\uparrow\rangle_{\mathrm{M}}$ and $|3,1\rangle_{\mathrm{M}} \equiv|\downarrow\rangle_{\mathrm{M}}$ for ${ }^{25} \mathrm{Mg}^{+}$ions. As in the main text, we use the symbol $\mathrm{B}(\mathrm{M})$ for ${ }^{9} \mathrm{Be}^{+}\left({ }^{25} \mathrm{Mg}^{+}\right)$ions, and label all states of ${ }^{9} \mathrm{Be}^{+}\left({ }^{25} \mathrm{Mg}^{+}\right)$ions with the subscript B (M) for the remainder of the supplementary materials. At our chosen magnetic field, the B qubits are first-order insensitive to magnetic field fluctuations. A Ramsey experiment with microwave pulses shows contrast higher than $90 \%$ after a Ramsey wait time of $1 \mathrm{~s}$; a Ramsey experiment with co-propagating laser carrier pulses shows similar results. The M qubits are first-order sensitive to magnetic field fluctuations with a linear coefficient of $\sim 430 \mathrm{kHz} \mathrm{mT}^{-1}$ (14). We stabilize the magnetic field by measuring the current applied to the magnetic field coils and feeding back on the voltage setpoint of the current supply to keep the measured current stable. Individual components of the algorithm are generally calibrated in experiments triggered on the $60 \mathrm{~Hz} \mathrm{AC}$ line, but these components are typically used at different phases of the $60 \mathrm{~Hz}$ AC line when applied in calibration experiments and in the QGT algorithm. To mitigate miscalibrations from this, we modulate the magnetic field current feedback setpoint with $60 \mathrm{~Hz}$ and $180 \mathrm{~Hz}$ signals (phases relative to the AC cycle and amplitudes determined experimentally) to reduce the magnetic field noise amplitude at these frequencies. We additionally synchronize the QGT algorithm with the $60 \mathrm{~Hz}$ AC line cycle to reduce decoherence caused by the remaining magnetic field noise at $60 \mathrm{~Hz}$ and its harmonics. These improvements result in a coherence time of $\sim 140 \mathrm{~ms}$ on the M qubit transition, measured independently in a Ramsey experiment using microwave pulses and in another Ramsey experiment using co-propagating 
Raman beams. Slow fluctuations in the ambient magnetic field noise lead to variations of the coherence time by up to $30 \%$ from day to day.

All laser beams used in this experiment are aligned to the ions at the laser interaction zone (LIZ), see Fig. 2 in the main text. At this location in the trap, both B and M ions experience micromotion along the direction the ions are aligned in (axial) with a modulation index of 2.67 for $\mathrm{B}$ ions and 1.08 for $\mathrm{M}$ ions (16). This micromotion arises due to trap imperfections. An electro-optic modulator (EOM) is placed in one of the B motion-sensitive Raman beamlines to compensate for this effect, which results in an effective modulation index of less than 0.1 for $\mathrm{B} ; \mathrm{M}$ axial micromotion is left uncompensated. All B motion-sensitive Raman transitions in this work are driven on the micromotion carrier instead of the second micromotion sideband as done in previous work in this trap $(14,30)$.

Crystal Preparation Prior to running experiments, we load a four-ion chain of two B ions and two $\mathrm{M}$ ions into a single well located at the LIZ with single-B-ion trap frequencies of 2.0 $\mathrm{MHz}$ (axial), $12.0 \mathrm{MHz}$ and $12.3 \mathrm{MHz}$ (radial, two orthogonal transverse directions to the crystal axis).

The initial order of ions in the ion crystal is random and can change on time-scales of minutes from background gas collisions. To set the order before each shot of the experiment, we increase the axial potential (to produce single-B-ion trap frequencies of $5.5 \mathrm{MHz}$ axial, $10.5 \mathrm{MHz}$ and $12.5 \mathrm{MHz}$ radial, but single-M-ion radial frequencies are considerably lower due to the inverse mass dependence of the radial pseudo-potential) while simultaneously laser cooling both species. In this case, the four-ion chain is deformed into an energetically favorable diamondshaped configuration where the two $\mathrm{M}$ ions are aligned radially and displaced symmetrically about the $z$-axis between the two B ions. Relaxing the trap back to the original confining potential brings the crystal to an ordered linear chain of $B_{1}-M_{1}-M_{2}-B_{2}$, with trap frequencies of 
1.4 MHz, $3.0 \mathrm{MHz}, 4.1 \mathrm{MHz}$, and $4.2 \mathrm{MHz}$ for the four axial normal modes.

Shuttling Operations To individually address the two pairs of B-and-M ions, the four-ion chain is separated into $\mathrm{B}_{1}-\mathrm{M}_{1}$ and $\mathrm{M}_{2}-\mathrm{B}_{2}$ in a process taking $570 \mu \mathrm{s}$ (Fig. $2 \mathrm{~A} \rightarrow 2 \mathrm{~B}$ ). The two ion pairs can then be separately transported to the LIZ by applying shuttling steps from a set of four primitives, each taking $230 \mu \mathrm{s}$. This set includes shuttling from the ion positions shown in Fig. 2B $\rightarrow 2 \mathrm{C}, 2 \mathrm{~B} \rightarrow 2 \mathrm{D}$, and their reversals. The full shuttling sequence is composed from these basic separation and transport operations $(16,17)$. While residing in the LIZ, the pairs of B-and-M ions are confined in a potential with trap frequencies of $2.1 \mathrm{MHz}$ and $4.5 \mathrm{MHz}$ for the two axial normal modes.

State Preparation and Detection To prepare for each experiment, the B and $\mathrm{M}$ ions are optically pumped to $\mid$ Bright $\rangle_{\mathrm{B}}$ and $\mid$ Bright $\rangle_{\mathrm{M}}$ respectively. The $\mathrm{M}$ ions are then transferred to the state $|\downarrow\rangle_{M}$ with microwave pulses. To detect their internal states, the ions are mapped from the computation basis to the measurement basis. The B ions are mapped individually with copropagating Raman beams from $|\uparrow\rangle_{B}$ to $\mid$ Bright $\rangle_{B}$ and from $|\downarrow\rangle_{B}$ to $\mid$ Dark $\rangle_{B}$, while the M ions are mapped with a combined scheme

$$
\begin{aligned}
& \left.|\downarrow\rangle_{\mathrm{M}} \stackrel{\text { laser }}{\longrightarrow}|2,+2\rangle_{\mathrm{M}} \stackrel{\text { microwave }}{\longrightarrow} \mid \text { Bright }\right\rangle_{\mathrm{M}} \\
& \left.|\uparrow\rangle_{\mathrm{M}} \stackrel{\text { laser }}{\longrightarrow}|3,-1\rangle_{\mathrm{M}} \stackrel{\text { microwave }}{\longrightarrow} \mid \text { Dark }\right\rangle_{\mathrm{M}}
\end{aligned}
$$

to maintain both individual addressing and high transfer efficiency ${ }^{1}$. After mapping out to the measurement basis, the internal states of the ions are distinguished by fluorescence detection for a duration of $330 \mu \mathrm{s}$ for $\mathrm{B}$ and $200 \mu \mathrm{s}$ for M ions. An EOM is placed in the B resonant beamline to approximately cancel the effect of axial micromotion. We obtain an approximately

\footnotetext{
${ }^{1}$ In contrast, all qubit operations for both species are implemented with laser pulses.
} 
Poissonian distribution of photon counts with a mean of roughly 30 counts for each individual $\mathrm{B}$ and $\mathrm{M}$ ion in the bright state, and average background counts of 1.5 and 0.8 for $\mathrm{B}$ and $\mathrm{M}$ respectively in the dark state. For conditional operations based on the $\mathrm{M}$ measurements, a threshold of 10 photon counts is set to distinguish $\mid$ Bright $\rangle_{M}$ from $\mid$ Dark $\rangle_{M}$ in a single measurement. Thresholds for the B measurements are discussed in the quantum process tomography section below.

Sideband Cooling At the beginning of the algorithm, we apply Doppler cooling (DC) on first $\mathrm{M}$ and then B ions followed by sideband cooling (SBC) on the B ions (31). The SBC sequence consists of continuous SBC applied sequentially to the four axial modes (32) followed by a short sequence of pulsed SBC (31), which cools the four-ion chain to an average motional quantum number $\bar{n}$ of less than 0.1 for the mode used to implement the MS gate and less than 0.3 for all the other axial modes. After separating the two B-and-M pairs and shuttling one pair of B-and-M ions to the LIZ (Fig 2C and 2D), Doppler cooling is only applied to the B ions to preserve the entanglement of the M qubits. The SBC on B ions cools both axial modes of the B-and-M pairs to an average occupation number $\bar{n}$ of less than 0.1 before applying two-qubit gate operations.

Logic Gates Two sets of Raman laser beams are used to implement the single-qubit and two-qubit operations in this experiment. For the $\mathrm{M}$ ions, a frequency-quadrupled diode laser ( $\sim 295 \mathrm{GHz}$ blue-detuned from the $\mathrm{S}_{1 / 2} \leftrightarrow \mathrm{P}_{3 / 2}$ transition) is used to produce all the frequencies necessary for driving co-propagating carrier transitions, motion-sensitive carrier transitions, and two-qubit Mølmer-Sørensen (MS) gates. Similarly, all laser beams for addressing the B ions are derived from a fiber laser system $\left(\sim 265 \mathrm{GHz}\right.$ red-detuned from the $\mathrm{S}_{1 / 2} \leftrightarrow \mathrm{P}_{1 / 2}$ transition). 
The co-propagating carrier pulses induce single-qubit rotations

$$
\hat{R}(\theta, \phi)=\left[\begin{array}{cc}
\cos \frac{\theta}{2} & -i \mathrm{e}^{-i \phi} \sin \frac{\theta}{2} \\
-i \mathrm{e}^{i \phi} \sin \frac{\theta}{2} & \cos \frac{\theta}{2}
\end{array}\right]
$$

where $\theta$ is the angle rotated and $\phi$ is the angle between the rotation axis (in the $x y$ plane) and the positive $x$-axis of the Bloch sphere.

We use a pair of M ions in the Bell state $\left|\Phi^{+}\right\rangle_{\mathrm{M}}=\frac{1}{\sqrt{2}}\left(|\uparrow \uparrow\rangle_{\mathrm{M}}+|\downarrow \downarrow\rangle_{\mathrm{M}}\right)$ as the entanglement resource for the teleported CNOT gate. This entangled pair is generated with an MS interaction applied only to the $\mathrm{M}$ ions in the four-ion chain. The gate is implemented on the out-of-phase mode at $3.0 \mathrm{MHz}$ with a gate duration of about $56 \mu \mathrm{s}$. The MS interaction implemented here does not generate a deterministic Bell state due to a slowly-fluctuating interferometric phase between the two arms of the motion-sensitive Raman beams.

To mitigate this, the MS pulse is surrounded with a pair of carrier $\pi / 2$ pulses using the same set of motion-sensitive laser beams, as shown in Fig. S2A, resulting in phase gates

$$
\hat{G}_{+}=\left[\begin{array}{cccc}
1 & 0 & 0 & 0 \\
0 & i & 0 & 0 \\
0 & 0 & i & 0 \\
0 & 0 & 0 & 1
\end{array}\right] \text { or } \hat{G}_{-}=\left[\begin{array}{cccc}
1 & 0 & 0 & 0 \\
0 & -i & 0 & 0 \\
0 & 0 & -i & 0 \\
0 & 0 & 0 & 1
\end{array}\right],
$$

that do not depend on the fluctuating interferometric phase $(14,19)$. The sign of the implemented phase gate depends on the sign of the MS detuning from the motional sidebands, phases of the surrounding single-qubit rotations, and in the case of mixed-species gates on the motion phases which define the direction of the ions' displacement in phase space $(14,19)$.

When constructing our $\mathrm{M}_{1}-\mathrm{M}_{2}$ Bell state, the MS detuning was chosen to be positive, and the two M ions share common single-qubit-rotation phases from global laser beams. This results in the phase gate $\hat{G}_{-}$, which is further surrounded with a pair of co-propagating carrier $\pi / 2$ pulses to construct the Bell-state-generating gate $\hat{F}$ used to entangle the two M ions (Fig. S2B). We further verify through a parity scan that the $\mathrm{M}_{1}-\mathrm{M}_{2}$ Bell state is $\left|\Phi^{+}\right\rangle_{\mathrm{M}}=\frac{1}{\sqrt{2}}\left(|\uparrow \uparrow\rangle_{\mathrm{M}}+|\downarrow \downarrow\rangle_{\mathrm{M}}\right)$ after applying $\hat{F}$ to $|\downarrow \downarrow\rangle_{\mathrm{M}}$. 
The CNOT gates within the B-and-M pairs are implemented with a mixed-species MS interaction on the in-phase mode at $2.1 \mathrm{MHz}(14)$. We produce a phase gate similarly as done for $\hat{F}$, but with two pairs of perpendicular Raman beams, each pair addressing only one species. This gives additional degrees of freedom in the form of a differential motion phase between species and different phases for the surrounding single-qubit rotations, resulting in ambiguity about the resulting phase gate. To ensure that we produce the right form of phase gate $\left(\hat{G}_{+}\right)$, we calibrate the phases of the single-qubit rotations by running a complete mixed-species CNOT gate (Fig. S2C) while looking for the appropriately conditioned bit flip on the target. Here, the $\mathrm{CNOT}_{\mathrm{B}_{1} \rightarrow \mathrm{M}_{1}}\left(\mathrm{CNOT}_{\mathrm{M}_{2} \rightarrow \mathrm{B}_{2}}\right)$ with $\mathrm{B}(\mathrm{M})$ as the control is constructed by surrounding the phase gate $\hat{G}_{+}$with additional co-propagating carrier $\pi / 2$ pulses on the M (B) ion.

The rotation

$$
\hat{R}_{\mathrm{Z}}(\alpha)=\left[\begin{array}{cc}
\mathrm{e}^{-i \alpha / 2} & 0 \\
0 & \mathrm{e}^{i \alpha / 2}
\end{array}\right]
$$

at the end of the pulse groups is implemented in software by changing the phase of subsequent single-qubit rotations for that qubit by $-\alpha$. This is possible because the two-qubit phase gates $\hat{G}_{ \pm}$commute with $\hat{R}_{Z}(\alpha)$ rotations.

Phase Tracking The experiment is performed with a direct digital synthesizer (DDS) running in the "absolute phase" mode, where the phase of the DDS is reset if a frequency or phase change is necessary. The phase evolution of the qubits in the laboratory frame is tracked on the experiment control computer by accounting for the free precession frequency $f_{0}$ of the qubits and the precise timing $t$ from the first pulse to the current pulse. To apply a rotation at phase $\phi$, the corresponding DDS is set to the phase $2 \pi \cdot f_{0} \cdot t+\phi+\phi_{0}$, where the additional offset $\phi_{0}$ takes into account the phase shifts induced by the AC-Stark effect and magnetic field gradient. This removes the requirement of tracking the phase of each individual qubit with an independent DDS. In this experiment, we calibrate the phase offset $\phi_{0}$ for each co-propagating carrier pulse 
with a separate Ramsey experiment. This could be avoided in future experiments by calibrating the AC-Stark shift and the magnetic-field-gradient-induced phase shift and calculating the required phases from these calibrations.

Drifts and Calibration To improve the long-term stability of our experiment, parameters such as pulse lengths and transition frequencies are re-calibrated during data acquisition to reduce the effect of slow drifts in laser output, beam pointing, and magnetic field strength. We observe that these experimental parameters drift significantly over time-scales of several minutes to hours, which affects the fidelity of the algorithm and all its components. For example, Fig. S3 shows the measured fidelity of the $\mathrm{M}_{1}-\mathrm{M}_{2}$ Bell state (without correcting for state preparation and measurement (SPAM) errors) over a period of four hours without recalibration. The fidelity of the Bell state drifts away from the value obtained after calibration. Therefore, to maintain all of the operations used at a nearly constant and high fidelity, running the algorithm is conditioned upon fulfilling several validation experiments (validators). The validators verify that parameters were sufficiently accurate within a predetermined time period before the algorithm is scheduled, otherwise re-calibration is triggered. Each validator is in turn conditioned on its dependencies. Consider the $\mathrm{B}$ co-propagating carrier rotation $\hat{R}(\pi / 2,0)$ as an example: with $\mathrm{B}$ ions initialized in $|\uparrow\rangle_{\mathrm{B}}$, the carrier $\pi / 2$ pulses are validated by observing that 1 ) the probability of four successive pulses bringing the ions back to $|\uparrow\rangle_{B}$ and 2) the probability of six successive pulses bringing the ions to $|\downarrow\rangle_{\mathrm{B}}$ are both higher than their pre-defined thresholds. Failing the validation will trigger recalibration of the pulse parameters (pulse duration, transition frequency, and phase offset between subsequent pulses) and validators of the pulse parameters' dependencies. Here, the calibration of the pulse parameters of the B co-propagating carrier rotations relies on the correct values of magnetic field and mapping pulses, which serve as the dependencies. 
Maintaining the fidelity of these operations at a consistent level significantly mitigates the effect of experimental drifts. As shown in Fig. S4, we track the fidelities of Bell states generated from the MS, $\hat{F}$ and CNOT gates while taking tomography data. The standard deviation of these measurements does not differ significantly from the uncertainty of the individual data points due to projection noise.

Quantum Process Tomography For the required informationally-complete set of measurements, the two B ions were prepared in the following 16 different combinations of input states by single-qubit rotations after initial state preparation to the $|\uparrow \uparrow\rangle_{\mathrm{B}}$ state:

$\begin{array}{llll}|\uparrow \uparrow\rangle_{\mathrm{B}} & |\uparrow \downarrow\rangle_{\mathrm{B}} & |\uparrow+\rangle_{\mathrm{B}} & |\uparrow r\rangle_{\mathrm{B}} \\ |\downarrow \uparrow\rangle_{\mathrm{B}} & |\downarrow \downarrow\rangle_{\mathrm{B}} & |\downarrow+\rangle_{\mathrm{B}} & |\downarrow r\rangle_{\mathrm{B}} \\ |+\uparrow\rangle_{\mathrm{B}} & |+\downarrow\rangle_{\mathrm{B}} & |++\rangle_{\mathrm{B}} & |+r\rangle_{\mathrm{B}} \\ |r \uparrow\rangle_{\mathrm{B}} & |r \downarrow\rangle_{\mathrm{B}} & |r+\rangle_{\mathrm{B}} & |r r\rangle_{\mathrm{B}}\end{array}$

where $|+\rangle_{\mathrm{B}}=\frac{1}{\sqrt{2}}\left(|\uparrow\rangle_{\mathrm{B}}+|\downarrow\rangle_{\mathrm{B}}\right)$ and $|r\rangle_{\mathrm{B}}=\frac{1}{\sqrt{2}}\left(|\uparrow\rangle_{\mathrm{B}}+i|\downarrow\rangle_{\mathrm{B}}\right)$. For each of these 16 inputs, the output states were measured along nine different combinations of measurement axes XX, XY, $\mathrm{XZ}, \mathrm{YX}, \mathrm{YY}, \mathrm{YZ}, \mathrm{ZX}, \mathrm{ZY}, \mathrm{ZZ}$, where a measurement along the Z axis was implemented by transferring to the measurement basis and performing fluorescence detection, and a measurement along the $\mathrm{X}$ or $\mathrm{Y}$ axis was performed similarly after a rotation $\hat{R}(\pi / 2,-\pi / 2)$ or $\hat{R}(\pi / 2,0)$, respectively.

We collected two full data sets for process tomography, and used dataset 1 to develop the final analysis protocol which was then applied without further modifications to dataset 2 . Process tomography is performed by preparing the informationally-complete set of input states $\left\{\rho_{k}\right\}_{k=1}^{16}$ listed above, applying the QGT algorithm to each input state, and measuring the two B ions in one of the 9 possible Pauli-product bases. Each Pauli-product basis constitutes a positive operator-valued measure (POVM) with 4 elements, corresponding to the detections of bright or dark states of the two ions. The family of $4 \times 9$ POVM elements $\left\{E_{l}\right\}_{l=1}^{36}$ obtained from the 
POVMs for the 9 Pauli-product bases is informationally complete for state tomography. In this section, we refer to running the algorithm with a single choice of state preparation and measurement basis as an experiment, and a single instance of input state preparation, QGT algorithm, and measurement as a trial. We assume perfect preparation of the input B states, but to account for the small overlap between photon-count distributions of the bright and dark states during detection, we model the POVM elements for individual ion measurements as a convex sum of projectors onto the bright and dark states. From a set of reference photon count histograms recorded periodically between experiments during the data acquisition, we infer the POVM elements by finding the thresholds and weights in the convex sum that maximize the likelihood of the observed reference histogram data (See Fig. S5). This method for inferring the POVM elements is a special case of the measurement tomography procedure described in (33).

Given a quantum process $\mathcal{E}$ applied to input state $\rho_{k}$, the probability of observing measurement outcome $E_{l}$ on a single trial is $\operatorname{Pr}\left(E_{l} \mid \rho_{k}\right)=\operatorname{Tr}\left(\mathcal{E}\left(\rho_{k}\right) E_{l}\right)$. The probability of observing all the recorded experimental data is given by the likelihood function:

$$
L(\mathcal{E})=\prod_{k l} \operatorname{Tr}\left(\mathcal{E}\left(\rho_{k}\right) E_{l}\right)^{n_{k l}}
$$

where $n_{k l}$ is the number of times outcome $E_{l}$ was observed when state $\rho_{k}$ was prepared. We estimate the process using the method of maximum likelihood (ML), which involves maximizing the $\log$-likelihood function $\mathcal{L}(\mathcal{E})=\ln (L(\mathcal{E}))$ over all two-qubit completely-positive tracepreserving (CPTP) maps $\mathcal{E}$. We use the Choi matrix representation (34), in which the process $\mathcal{E}$ is represented by the $d^{2}$-by- $d^{2}$ density matrix $\chi=(I \otimes \mathcal{E})\left(\left|\Phi^{+}\right\rangle\left\langle\Phi^{+}\right|\right)$, where $d=4$ is the dimension of the Hilbert space, $\left|\Phi^{+}\right\rangle=\frac{1}{\sqrt{d}} \sum_{i=0}^{d-1}|i\rangle|i\rangle$ is a maximally entangled state between two copies of the Hilbert space, and $I$ is the identity matrix. In terms of the Choi matrix, the action of the process on an input state is given by $\mathcal{E}\left(\rho_{k}\right)=d \operatorname{Tr}_{1}\left(\chi\left(\rho_{k}^{\top} \otimes I\right)\right)$, where $\operatorname{Tr}_{1}$ denotes a partial trace over the first subsystem and ${ }^{\top}$ denotes transposition. The log-likelihood function 
is then given by

$$
\mathcal{L}(\mathcal{E})=\sum_{k l} n_{k l} \ln \left(d \operatorname{Tr}\left(\chi\left(\rho_{k}^{\top} \otimes E_{l}\right)\right)\right)
$$

We use the " $R \rho R$ " algorithm for processes (35) to find the Choi matrix $\hat{\chi}$ that maximizes the log-likelihood function. Throughout this section, a "hat" placed above the symbol for a physical quantity is used to denote a statistical estimate of that quantity. The entanglement fidelity (36) with respect to $U=\mathrm{CNOT}$ is defined to be

$$
F(\mathcal{E}, U)=\left\langle\Phi^{+}\left|\left(I \otimes U^{\dagger}\right) \chi(I \otimes U)\right| \Phi^{+}\right\rangle
$$

From the ML-estimated process we obtain the ML entanglement fidelities $\hat{F}_{M L}=0.858$ for dataset 1 and $\hat{F}_{M L}=0.851$ for dataset 2 .

We obtain confidence intervals for the process fidelity using a parametric bootstrap method. Associated with each confidence interval is a confidence level. A confidence interval is defined as follows: in an ensemble of identically analyzed data sets (following the assumed model), one expects the frequency, with which the true value of the fidelity for any given data set will lie within that data set's confidence interval, to equal the confidence level. The ML estimate $\hat{\chi}$ is used to simulate 2000 synthetic data sets, and ML is run on each synthetic data set, producing a distribution of bootstrapped fidelities shown in Fig. S6. We then compute the basic bootstrap confidence interval (23), which is defined as follows. Let $\hat{F}_{M L}$ be the ML estimated fidelity, and for $\alpha \in[0,1]$, let $f_{\alpha}$ be the fidelity value corresponding to the $(100 \alpha)^{\text {th }}$ percentile of the bootstrapped distribution. Then the $100(1-2 \alpha)$ percent confidence interval is $\left[2 \hat{F}_{M L}-\right.$ $\left.f_{(1-\alpha)}, \quad 2 \hat{F}_{M L}-f_{\alpha}\right]$. The endpoints of the confidence interval are obtained by reflecting the upper and lower percentile values of the bootstrapped distribution about the ML estimate. This results in a confidence interval that approximately corrects for bias in the ML estimate (see Fig. S6). Using this method, we obtain $95 \%$ confidence intervals of $[0.852,0.878]$ for dataset 1 and $[0.845,0.872]$ for dataset 2 . Confidence intervals for other confidence levels are in Table S2. 
As a consistency check against the ML fidelity estimate, we also constructed a linear esti-

mator $\hat{F}_{L}$ that computes the fidelity directly from the observed frequencies: $\hat{F}_{L}=\sum_{k l} a_{k l} f_{k l}$, where $f_{k l}$ is the observed frequency of seeing outcome $E_{l}$ given state preparation $\rho_{k}$. To derive the coefficients $a_{k l}$, we first rewrite Eq. 8 as

$$
F(\mathcal{E}, U)=\frac{1}{d^{2}} \sum_{i, j=0}^{d-1}\left\langle i\left|U^{\dagger} \mathcal{E}(|i\rangle\langle j|) U\right| j\right\rangle .
$$

Since the set of input states and the set of POVM elements each form a complete operator basis, we can make the following expansions:

$$
\begin{gathered}
|i\rangle\langle j|=\sum_{k} b_{k}^{(i j)} \rho_{k} \\
U|j\rangle\langle i| U^{\dagger}=\sum_{l} c_{l}^{(j i)} E_{l}
\end{gathered}
$$

Plugging back into Eq. 9, we get

$$
\begin{aligned}
F(\mathcal{E}, U) & =\frac{1}{d^{2}} \sum_{i j} \sum_{k l} b_{k}^{(i j)} c_{l}^{(j i)} \operatorname{Tr}\left(E_{l} \mathcal{E}\left(\rho_{k}\right)\right) \\
& =\frac{1}{d^{2}} \sum_{i j} \sum_{k l} b_{k}^{(i j)} c_{l}^{(j i)} \operatorname{Pr}\left(E_{l} \mid \rho_{k}\right)
\end{aligned}
$$

and therefore $a_{k l}=\sum_{i j} b_{k}^{(i j)} c_{l}^{(j i)}$. It remains to determine the coefficients $b_{k}^{(i j)}$ and $c_{l}^{(j i)}$. The set $\left\{E_{l}\right\}_{l}$ is an overcomplete basis, so there is a degenerate set of solutions to Eq. 11 . We proceed as follows: let $\left.\left|\rho_{k}\right\rangle\right\rangle$ be a vectorization of $\rho_{k}$ and define the superoperator $\left.\mathcal{S}=\sum_{k}\left|\rho_{k}\right\rangle\right\rangle\left\langle\left\langle\rho_{k}\right|\right.$. Then the vectorized dual basis density matrix $\tilde{\rho_{k}}$ corresponding to $\rho_{k}$ is defined by $\left.\left.\left|\tilde{\rho_{k}}\right\rangle\right\rangle=\mathcal{S}^{-1}\left|\rho_{k}\right\rangle\right\rangle$. The dual basis POVM elements $\tilde{E}_{l}$ are defined analogously. Let $\left.|i j\rangle\right\rangle$ be a vectorization of $|i\rangle\langle j|$. Then

$$
\begin{aligned}
|i j\rangle\rangle & \left.=\mathcal{S S}^{-1}|i j\rangle\right\rangle \\
& \left.=\sum_{k}\left|\rho_{k}\right\rangle\right\rangle\left\langle\left\langle\rho_{k}\left|\mathcal{S}^{-1}\right| i j\right\rangle\right\rangle \\
& \left.=\sum_{k}\left|\rho_{k}\right\rangle\right\rangle\left\langle\left\langle\tilde{\rho}_{k} \mid i j\right\rangle\right\rangle,
\end{aligned}
$$


and therefore a solution to Eq. 10 is given by $b_{k}^{(i j)}=\left\langle\left\langle\tilde{\rho}_{k} \mid i j\right\rangle\right\rangle=\left\langle j\left|\tilde{\rho_{k}}\right| i\right\rangle$. Similarly, a solution to Eq. 11 is given by $c_{l}^{(j i)}=\left\langle i\left|U^{\dagger} \tilde{E}_{l} U\right| j\right\rangle$. Plugging into Eq. 12 and after some simplification, we find that the coefficients in the linear estimator are given by

$$
a_{k l}=\frac{1}{d^{2}} \operatorname{Tr}\left(U \tilde{\rho}_{k} U^{\dagger} \tilde{E}_{l}\right)
$$

The linear fidelity estimator is a consistent and unbiased estimator, meaning that $\hat{F}_{L}$ converges to $F(E, U)$ in the limit of infinite trials per experiment and the expectation of $\hat{F}_{L}$ is $F(E, U)$. However, $\hat{F}_{L}$ has a larger variance than $\hat{F}_{M L}$, in part due to effects that occur at the boundary of quantum state space. We generate 2000 non-parametric bootstrapped data sets to obtain error bars on the linear fidelity estimate. The distribution of bootstrapped linear fidelity estimates is shown in Fig. S6. As $\hat{F}_{L}$ is unbiased, its value for the experimental data approximately equals the mean of the bootstrapped distribution. Therefore the basic bootstrap confidence interval matches the interval between the corresponding quantiles of the distribution. We obtain a $95 \%$ basic bootstrap confidence interval of $[0.845,0.888]$ for $\hat{F}_{L}$, which contains the ML entanglement fidelity. The results from the ML estimation and the linear estimation of the two data sets are summarized in Table S2.

Pauli Transfer Matrix The ML-estimated process shown in Fig. 3 of the main text is in the Pauli transfer matrix representation. Let $\left\{P_{i}\right\}_{i=0}^{d^{2}-1}$ be an operator basis of $n$-qubit Pauli operators, where $d=2^{n}$, and $P_{0}=I$. Then the Pauli transfer matrix $\mathcal{T}$ has entries $\mathcal{T}_{i j}=$ $\frac{1}{d} \operatorname{Tr}\left(P_{i} \mathcal{E}\left(P_{j}\right)\right)$. The CPTP constraint on $\mathcal{E}$ results in the properties that $\mathcal{T}_{00}=1, \mathcal{T}_{0 j}=0$ for $j>0$, and $-1 \leq \mathcal{T}_{i j} \leq 1$ for all $i, j$. The Pauli transfer matrix elements are related to the Choi matrix by

$$
\mathcal{T}_{i j}=\operatorname{Tr}\left(\chi\left(P_{j}^{\top} \otimes P_{i}\right)\right)
$$


Likelihood Ratio Test As a further consistency check, we perform a likelihood ratio test to investigate whether a CPTP map acting on a two-qubit state space is a good model for the observed data. The general problem of deciding between models to fit data is called model selection. For a recent reference on the use of model selection in quantum tomography, see (37). A model $\mathcal{M}$ is a parametrized set of probability distributions. Given two nested models $\mathcal{M}_{0} \subset \mathcal{M}_{1}$, the likelihood ratio test allows one to decide whether or not to reject the null hypothesis that the observed data is sampled from a distribution in the model $\mathcal{M}_{0}$. In our case, let $\mathcal{M}_{0}$ denote the model of all probability distributions that could result from the application of a single CPTP map $\mathcal{E}$ on each trial during the process tomography protocol. Let $\mathcal{M}_{1}$ denote the fully unrestricted model, that is, the set of all 144 independent probability distributions (one distribution for each combination of state preparation and measurement basis) on 4 elements. The log-likelihood ratio statistic $\lambda$ is defined by

$$
\lambda=2\left(\mathcal{L}\left(\mathcal{M}_{1}\right)-\mathcal{L}\left(\mathcal{M}_{0}\right)\right)
$$

where $\mathcal{L}\left(\mathcal{M}_{0}\right)=\max _{\mathcal{E}}(\mathcal{L}(\mathcal{E}))$ is the maximum of the log-likelihood function defined in Eq. 7, and $\mathcal{L}\left(\mathcal{M}_{1}\right)=\sum_{k l} n_{k l} \ln \left(p_{k l}\right)$ is the maximum log-likelihood of the fully unrestricted model given the observed data.

Likelihood ratio tests often assume that $\lambda$ has a chi-squared distribution, but because of boundary effects, this assumption is typically not true in quantum tomography experiments (37). Rather than using a chi-squared distribution, our likelihood ratio test compares the value of $\lambda$ computed from the experimental data with the distribution of values for $\lambda$ obtained from the bootstrapped data sets. Roughly, if the experimental value of $\lambda$ is near the center of the bootstrapped distribution, then there is no statistical evidence for rejecting $\mathcal{M}_{0}$. We quantify model discrepancy as $(\lambda-\bar{\lambda}) / \sigma$, where $\lambda$ is obtained from the original data, and $\bar{\lambda}$ and $\sigma$ are the mean and standard deviation of the log-likelihood ratio statistic from the bootstrapped data sets. 
The results of the likelihood ratio test are shown in Fig. S7 . We observe model discrepancy at the level of 7.1 and 3.6 standard deviations for dataset 1 and 2, respectively. This indicates that the data is inconsistent with the null model $\mathcal{M}_{0}$ : the application of a single CPTP map to the specified input states followed by measurement by the inferred POVM does not fit the data as well as the fully unrestricted model. Such a discrepancy could be caused by context dependence, that is, a systematic dependence of the applied process on external variables (38).

A known systematic error present in the experiment is the drifts of Rabi rates for single-qubit rotations on the $\mathrm{B}$ ions. To investigate whether such drifts may be responsible for the observed model discrepancy, we simulated a data set with correlated over-rotation errors on the state preparation and measurement pulses as well as the single-qubit rotations when implementing the CNOT gates on B-and-M pairs shown in Fig. S2C. The magnitudes of the errors in the simulation drift according to the pattern observed in Fig. S8. A likelihood ratio test on the simulated data set yields a discrepancy at the level of 4.6 standard deviations, as shown in Fig. S7. We conclude that a large portion of the model discrepancy observed in the experimental data can be explained by drifts in the rotation angles.

To verify the existence of rotation-angle drifts for single-qubit rotations on B ions in the experimental setup, we performed a separate investigation in which we monitored the population of B ions after applying various numbers of $\pi / 2$ pulses as shown in Fig. S8A. The deviation from the starting population was converted to fractional changes in rotation angles $\delta \theta / \theta$ (Fig. S8B), where $\theta$ is the target pulse area, and $\delta \theta$ is the deviation of the actual pulse area from the target value. A maximum fractional change of up to $4 \%$ was observed over a period of 4 hours in the measurement shown. The drifts are mainly caused by the relative power fluctuation between the two spatially overlapped laser beams used to drive the co-propagating carrier, while the total power of the two is actively stabilized. The power ratio between the two beams was about $1: 3$ at the time of the measurement. Balancing the power ratio between 
the two beams reduced the drifts of Rabi rates, but the drifts at the percentage level remained. This can be improved by actively stabilizing the beat-note amplitude of the two beams in future experiments.

The likelihood ratio test helped identify a time-varying experimental imperfection that caused the experiment to depart from our model of a static process matrix. We believe this illustrates the importance of performing model consistency checks in addition to tomography when characterizing quantum processes.

Depolarizing Error Model We use a depolarizing model to estimate the total error of the QGT process (referred to as the algorithm in the following). Assuming a depolarizing error $\epsilon_{i}$ for a constituent process $\hat{U}_{i}$ of the algorithm, the density matrix after the process becomes

$$
\rho \rightarrow\left(1-\epsilon_{i}\right) \hat{U}_{i} \rho \hat{U}_{i}^{\dagger}+\epsilon_{i} \cdot \hat{I} / d_{i}
$$

where $d_{i}$ is the dimension of the Hilbert space for the process. Stepping through the full algorithm and applying a depolarizing error with the same magnitude as experimentally determined for each constituent process (which may or may not be depolarizing in reality) allows us to compute an approximate density matrix after the complete QGT process, and therefore derive the process matrix of this model process and its fidelity with respect to an ideal CNOT.

The error sources we consider here are SPAM errors on each qubit, the gate errors of each composite gate (Fig. S2), the error due to decoherence of the M ions, and depumping errors induced by stray resonant light.

The SPAM errors for the individual qubits are taken from SPAM diagnosis experiments (see Fig. S9A and S9B) interleaved with the tomography measurements. In the SPAM diagnosis experiments, we find the probability $\epsilon_{X, \uparrow}$ of measuring an ion in $|\downarrow\rangle_{X}$ after preparing it in the $|\uparrow\rangle_{X}$ state, where $X=\mathrm{B}_{1}, \mathrm{~B}_{2}, \mathrm{M}_{1}$, or $\mathrm{M}_{2}$. We also find the probability $\epsilon_{X, \downarrow}$ of measuring an ion in $|\uparrow\rangle_{X}$ after preparing it in the $|\downarrow\rangle_{X}$ state. The "Map In" and "Map Out" pulses for these 
measurements are described in section State Preparation and Detection. We use the mean value $\bar{\epsilon}_{X}=\frac{\epsilon_{X, \uparrow}+\epsilon_{X, \downarrow}}{2}$ to estimate the SPAM errors for each qubit in the QGT sequence. In addition, we also measured the SPAM errors of two $\mathrm{M}$ ions in a static well, using only microwave pulses for "Map In" and "Map Out". These results are listed in Tab. S3 and used later to account for errors in individual gates.

We use the Bell-state infidelities from each composite two-qubit gate with the contribution from SPAM errors subtracted as a representative estimate of their process infidelities. When estimating the Bell-state infidelity by measuring qubit populations and the contrast of parity oscillations (39), each qubit contributes an amount $\epsilon_{X \text {,Bell }}=\frac{3}{2} \bar{\epsilon}_{X}$ to the observed Bell-state infidelity (40). After subtracting the contribution from SPAM errors, we estimate an infidelity of $0.040(9)$ for the Bell-state-generating gate $\hat{F}$, and $0.030(9)$ and $0.03(1)$ for the mixed-species CNOT gates.

The error from $\mathrm{M}$ ion decoherence is estimated from the coherence time of a Ramsey experiment on a single $\mathrm{M}$ ion. Here we account for the fact that the two $\mathrm{M}$ ions are in the Bell state for a duration of $4.2 \mathrm{~ms}$ and that a coherent superposition needs to be preserved in $\mathrm{M}_{2}$ for a further $3.6 \mathrm{~ms}$. We model this decoherence error as equivalent to the contrast reduction in the Ramsey sequence on a single $\mathrm{M}$ ion with a wait time of $2 \times 4.2+3.6=12 \mathrm{~ms}$. From the $1 / e$ coherence time of $140(30) \mathrm{ms}$, we estimate the error from decoherence of the two M ions in the algorithm to be $0.007(3)$.

We further consider the errors from stray-resonant-light-induced depumping on ions outside of the LIZ. This is quantified as the difference between two SPAM experiments. A reference experiment measures the SPAM errors for one pair of B-and-M ions by shuttling the pair to the LIZ and performing the measurement there (Fig. S9A,B). In a separate experiment, we perform shuttling, cooling, state preparation, and detection in the same order as in the QGT experiment to measure the SPAM error plus the stray-light-induced depumping error (Fig. S9C). 
The difference of the two experiments is taken as the depumping error. We obtain errors of 0.011 (4) for $\mathrm{M}_{1}$ (due to the detection of $\mathrm{M}_{2}$ ) and 0.012 (3) for $\mathrm{B}_{2}$ (due to cooling and repumping of $\left.\mathrm{B}_{1}\right)$.

Based on the following assumptions and estimates, we can reject the hypothesis that the stray-resonant-light-induced depumping errors are induced solely by light scattering from the ions in the LIZ. Assuming a scattering rate of $R_{1}=\gamma_{\mathrm{M}} / 2$ for the $\mathrm{M}_{1}$ ion at the LIZ from a detection pulse with a duration of $\tau=200 \mu$ s, the $\mathrm{M}_{2}$ ion away from the LIZ experiences an intensity of $I_{2} \simeq \frac{\gamma_{\mathrm{M}}}{2} \cdot \frac{h c}{\lambda_{\mathrm{M}} \cdot 4 \pi l^{2}}$ from the scattering, where $\gamma_{\mathrm{M}}$ is the decay rate of the upper state of M ions, $h$ is the Planck's constant, $c$ is the speed of light, $\lambda_{\mathrm{M}}$ is the wavelength of $\mathrm{M}$ resonant light, and $l=340 \mu \mathrm{m}$ is the smallest distance between two minima of the double well. The $\mathrm{M}_{2}$ ion will scatter at a rate $R_{2}=\frac{I_{2}}{h c / \lambda_{\mathrm{M}}} \sigma_{\mathrm{M}}$ where $\sigma_{\mathrm{M}} \simeq \frac{3}{2 \pi} \lambda_{\mathrm{M}}^{2}$ is the absorption cross section of M ions at resonance. This implies a scattering probability of about $6 \times 10^{-4}$ for a detection period of $200 \mu \mathrm{s}$, which is much lower than the observed error rate of approximately one percent.

Depumping errors on the order of one percent indicate that on average at least 0.01 photons are scattered in one experiment due to fluorescence detection at the LIZ. In comparison, $\sim 1000$ resonant photons are scattered by an ion in the bright state in the LIZ. This is approximately equivalent to an intensity ratio of $\sim 0.01 / 1000=10^{-5}$ between the resonant light intensity outside of the LIZ compared to the light intensity in the LIZ. Reaching the goal of $10^{-4}$ error rates for practical fault tolerant quantum error correction would then require an intensity ratio of $\sim 10^{-4} / 1000=10^{-7}$. This presents a significant engineering requirement in future ion traps, but one that could be removed by completely eliminating the use of qubit resonant light and instead restricting all resonant operations to separate species ancillas (14). After any previous resonant interaction of a nearby ancilla ion has taken place, affected ancillas can be prepared again before use in the algorithm.

Inserting all errors from Table 1 in the main text as depolarizing errors into the model, we 
derive a total infidelity of $0.12(1)$ for the QGT algorithm. We derive the uncertainty of the total infidelity by applying a bootstrap to the depolarizing model. The fidelity determined from the depolarizing model is near the upper limits of the $95 \%$ confidence intervals obtained by both ML and linear estimation, indicating that the major error sources are reflected in this simplified error propagation model.

Other error sources, such as drifting experimental parameters, are not included in the depolarizing model. In addition, the drifts ins preparation and measurement pulses can cause bias in the estimate of the process fidelity. For example, we determine by simulation that rotationangle drifts of single-qubit rotations on B ions, uniformly distributed up to $5 \%$, lead to an under-estimation of process fidelity by $\sim 1 \%$. 


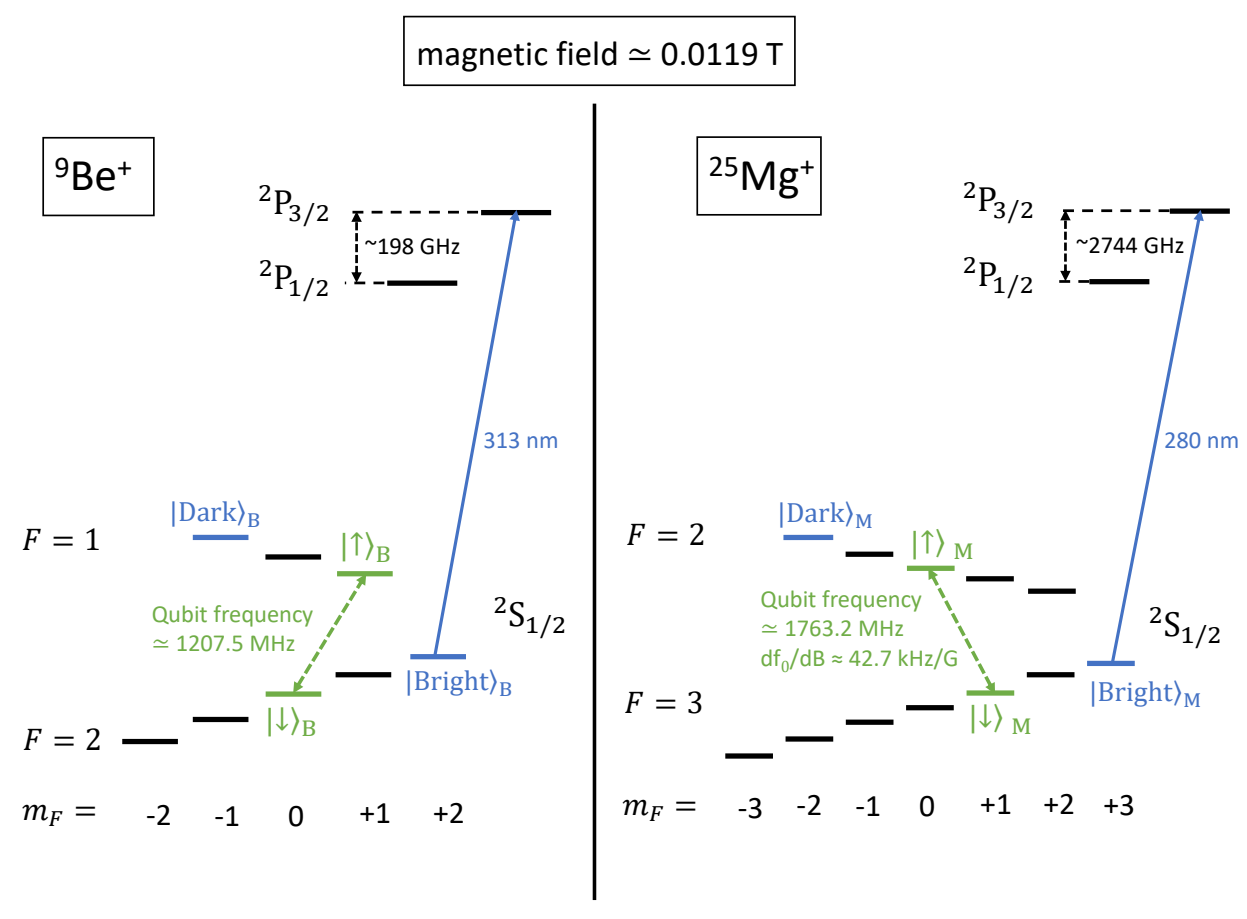

Figure S1: Relevant level structures of beryllium and magnesium ions. The ${ }^{9} \mathrm{Be}^{+}$qubits are encoded in the computation basis $|\uparrow\rangle_{\mathrm{B}}$ and $|\downarrow\rangle_{\mathrm{B}}$ and mapped out to the measurement basis $\mid$ Bright $\rangle_{\mathrm{B}}$ and $\mid$ Dark $\rangle_{\mathrm{B}}$ respectively for fluorescence detection with $313 \mathrm{~nm}$ light resonant with the ${ }^{2} \mathrm{~S}_{1 / 2} \mid$ Bright $\rangle_{\mathrm{B}} \leftrightarrow{ }^{2} \mathrm{P}_{3 / 2}|3,3\rangle_{\mathrm{B}}$ transition. The ${ }^{25} \mathrm{Mg}^{+}$qubits are encoded in the computation basis $|\uparrow\rangle_{\mathrm{M}}$ and $|\downarrow\rangle_{\mathrm{M}}$ and mapped out to the measurement basis $\mid$ Dark $\rangle_{\mathrm{M}}$ and $\mid$ Bright $\rangle_{\mathrm{M}}$ respectively for fluorescence detection with $280 \mathrm{~nm}$ light resonant with the ${ }^{2} \mathrm{~S}_{1 / 2}|\mathrm{Bright}\rangle_{\mathrm{M}} \leftrightarrow$ ${ }^{2} \mathrm{P}_{3 / 2}|4,4\rangle_{\mathrm{M}}$ transition. (See text) 
(A)

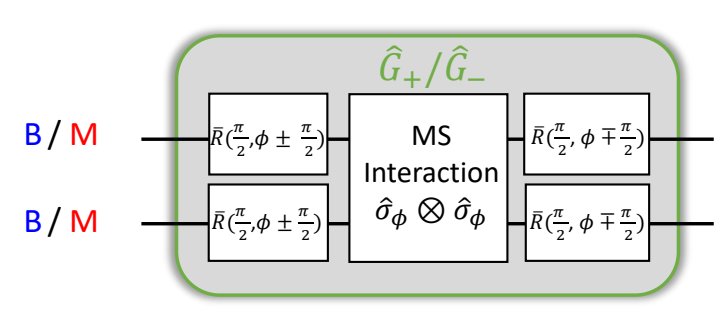

(B)

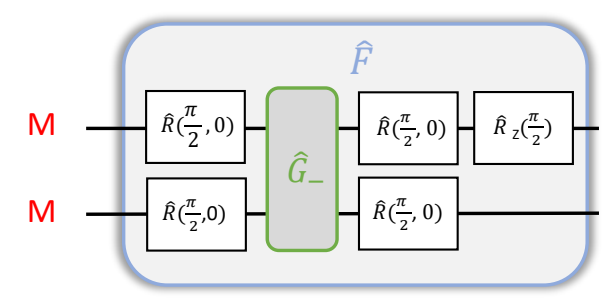

(C)

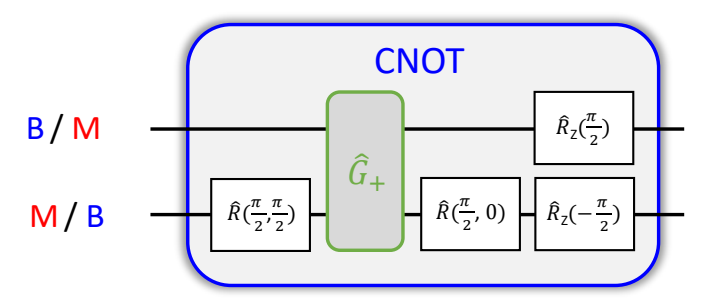

Figure S2: Composite gates. (A) Phase gates $\hat{G}_{ \pm}$are constructed by surrounding the MS pulse with single-qubit rotations $\bar{R}$, all driven by motion-sensitive Raman beams. The notation $\bar{R}$ distinguishes itself from motion-insensitive single-qubit rotations $\hat{R}$ driven by co-propagating Raman beams. The resulting operation does not depend on the uncontrolled interferometric phase of the Raman beam sets as long as this phase is constant during the entire $\hat{G}_{ \pm}$gate implementation. The sign of the implemented phase gate depends on the sign of MS detuning from motional sidebands, phases of surrounding single-qubit rotations, and motion phases in the case of mixed-species gates. (B) The $\mathrm{M}_{1}-\mathrm{M}_{2}$ Bell-state-generating gate $\hat{F}$ is constructed by surrounding $\hat{G}_{-}$with co-propagating $\pi / 2$ pulses and phase-shifting one of the qubits by $\pi / 2$ at the end. The sequence generates the $\mathrm{M}_{1}-\mathrm{M}_{2}$ Bell state $\left|\Phi^{+}\right\rangle_{\mathrm{M}}=\frac{1}{\sqrt{2}}\left(|\uparrow \uparrow\rangle_{\mathrm{M}}+|\downarrow \downarrow\rangle_{\mathrm{M}}\right)$ from the $|\downarrow \downarrow\rangle_{\mathrm{M}}$ state. (C) The CNOT gate is constructed by surrounding $\hat{G}_{+}$with co-propagating $\pi / 2$ pulses on the target qubit and phase shifting the control and the target qubit by $\pi / 2$ and $-\pi / 2$ respectively. 


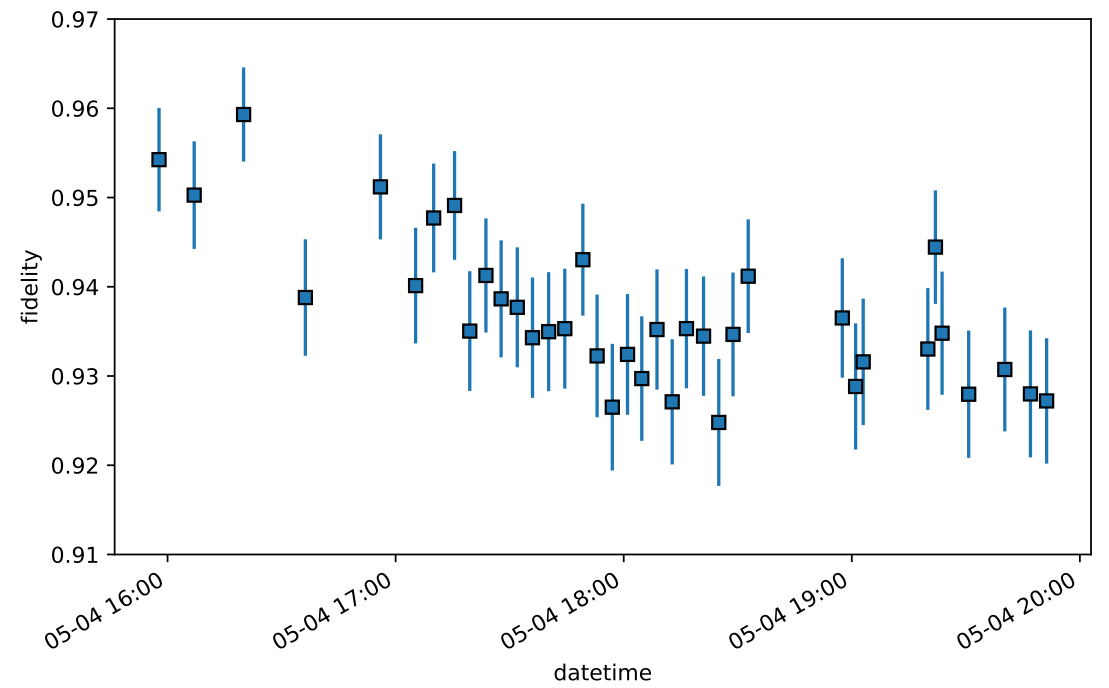

Figure S3: Drifts of gate fidelity. The fidelity of the $\mathrm{M}_{1}-\mathrm{M}_{2}$ Bell state generated by $\hat{F}$ was measured continuously over a period of four hours without re-calibration of experimental parameters, showing the drift away from the initial value over time. The exact direction and rate of the drift varies from run to run. The data shown here was taken on May 04, 2018. The gaps near 16:00 and 18:30 are caused by reloading the ions. 

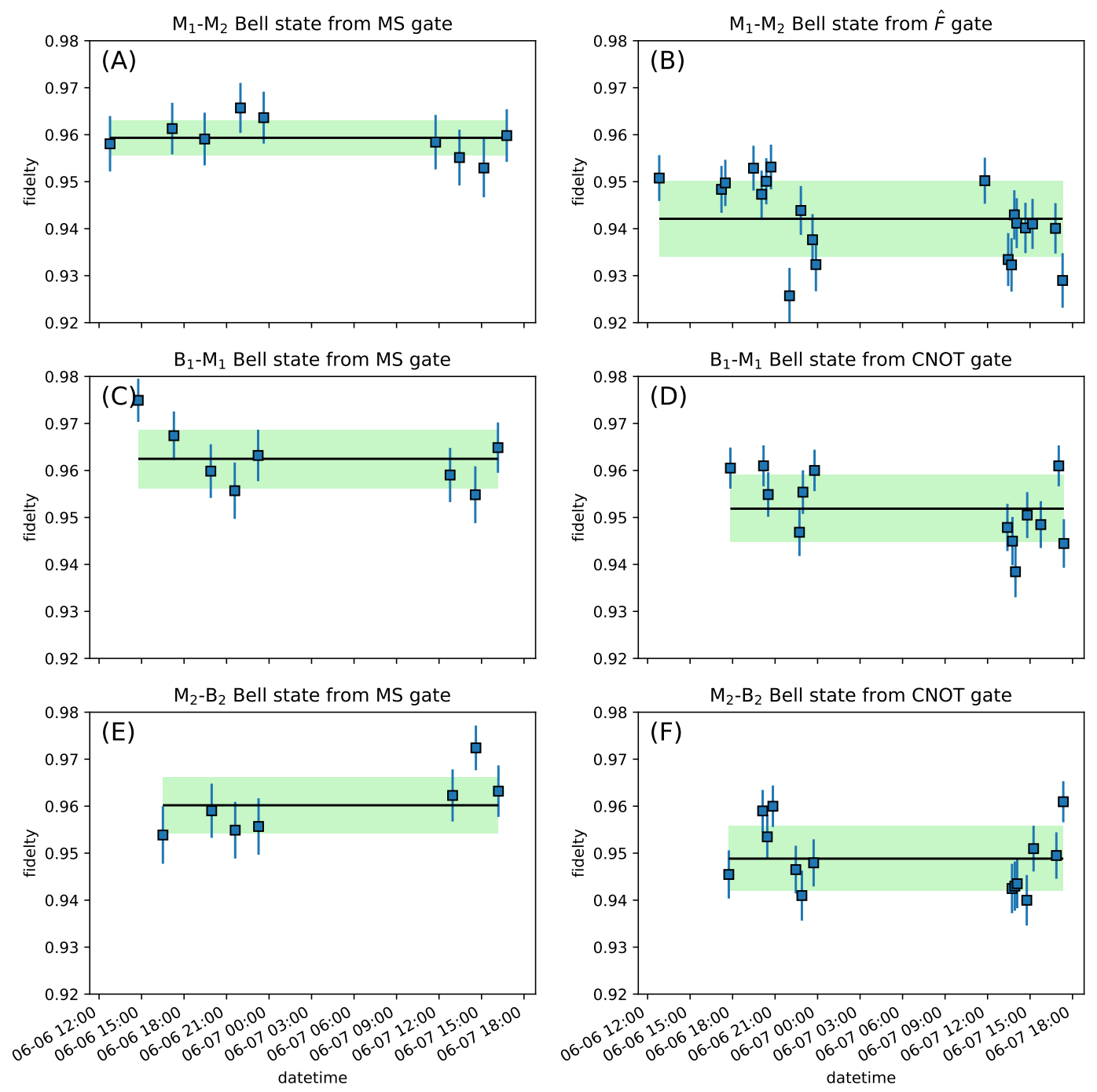

Figure S4: Bell-state fidelities. Bell-state fidelity measurements interspersed while taking full process tomography data on the teleported CNOT, without correcting for SPAM. The measurement spans over two days from June 6 to June 7, 2018. (A) $M_{1}-M_{2}$ Bell state from the MS gate. (B) $\mathrm{M}_{1}-\mathrm{M}_{2}$ Bell state from $\hat{F}$. For (A) and (B), microwave pulses are used for mapping into and out of the computation basis. (C) $B_{1}-M_{1}$ Bell state from MS gate. (D) $B_{1}-M_{1}$ Bell state from CNOT gate. (E) $\mathrm{M}_{2}-\mathrm{B}_{2}$ Bell state from MS gate. (F) $\mathrm{M}_{2}-\mathrm{B}_{2}$ Bell state from CNOT gate. Squares: fidelity data. Solid black line: mean fidelity. Green band: standard deviation of the data points. The error bars on the fidelity data are the uncertainties of each fidelity measurement due to projection noise. 

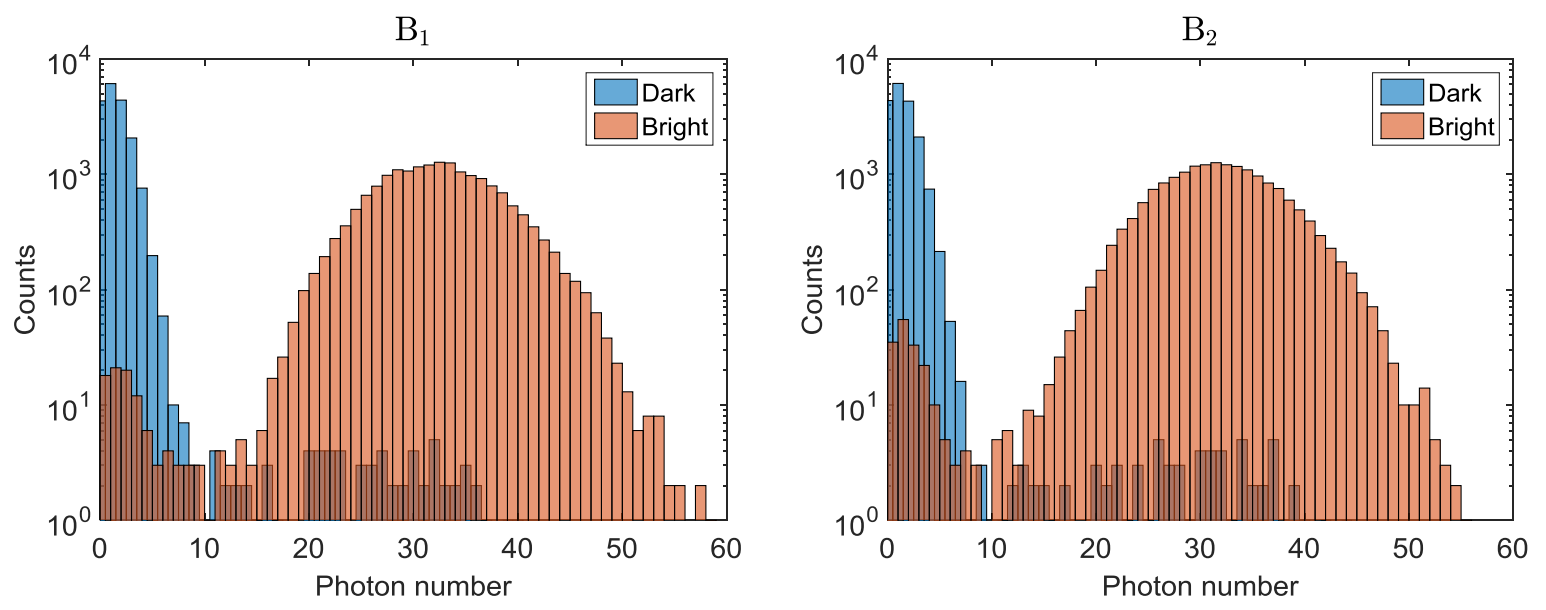

Figure S5: Reference histograms. Histograms of photon number counts when B ions are prepared in dark and bright states. Histograms are obtained from measurements taken during data acquisition. Photon count thresholds of 8 and 9 for $\mathrm{B}_{1}$ and $\mathrm{B}_{2}$, respectively, are determined to minimize the probability of misclassification. The reference histograms are used to infer the single-qubit POVM element $E_{+}$corresponding to observing a photon number greater than the threshold: $E_{+}=(1-p) \mid$ Bright $\rangle_{\mathrm{B} \text { B }}\langle\text { Bright }|+p| \text { Dark }\rangle_{\mathrm{B} \text { B }}\langle$ Dark $|$. We obtain $p=0.0090$ and $p=0.0134$ for $\mathrm{B}_{1}$ and $\mathrm{B}_{2}$, respectively. 


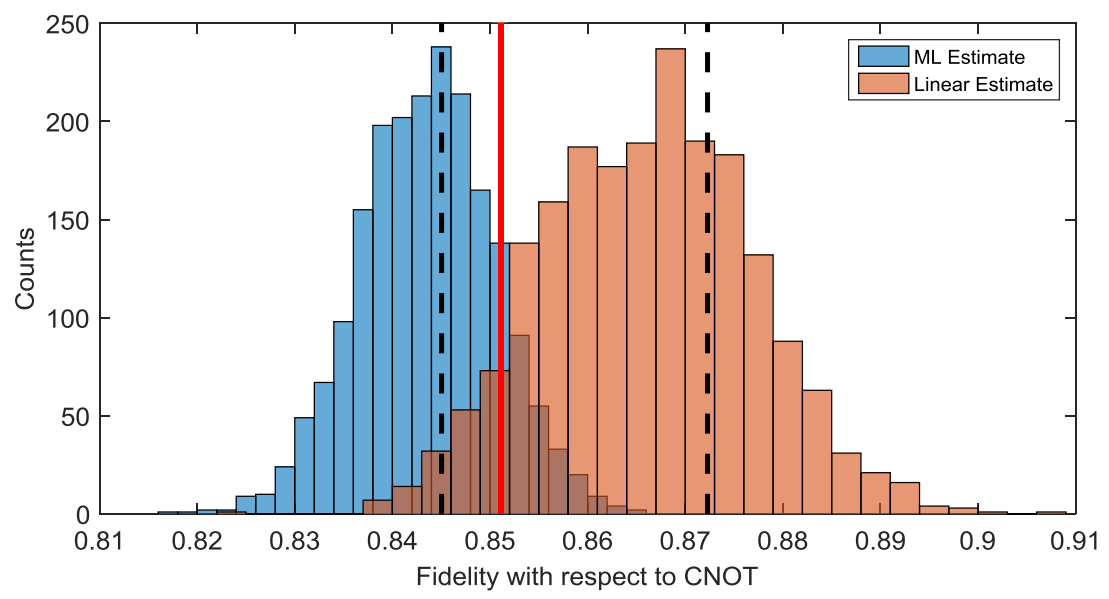

Figure S6: Histograms of bootstrapped fidelity distributions. The blue (left) histogram displays the distribution of process fidelities obtained from running ML on 2000 parametric bootstrapped data sets generated from the ML estimated process $\hat{\chi}$ of dataset 2 . The red solid vertical line indicates the process fidelity of $\hat{\chi}$. The black vertical dashed lines mark the boundary of a 95\% confidence interval computed via the basic bootstrap method. The orange (right) histogram displays the distribution of fidelities obtained from applying the linear fidelity estimator $\hat{F}_{L}$ to 2000 non-parametric resamples of the experimental data set.
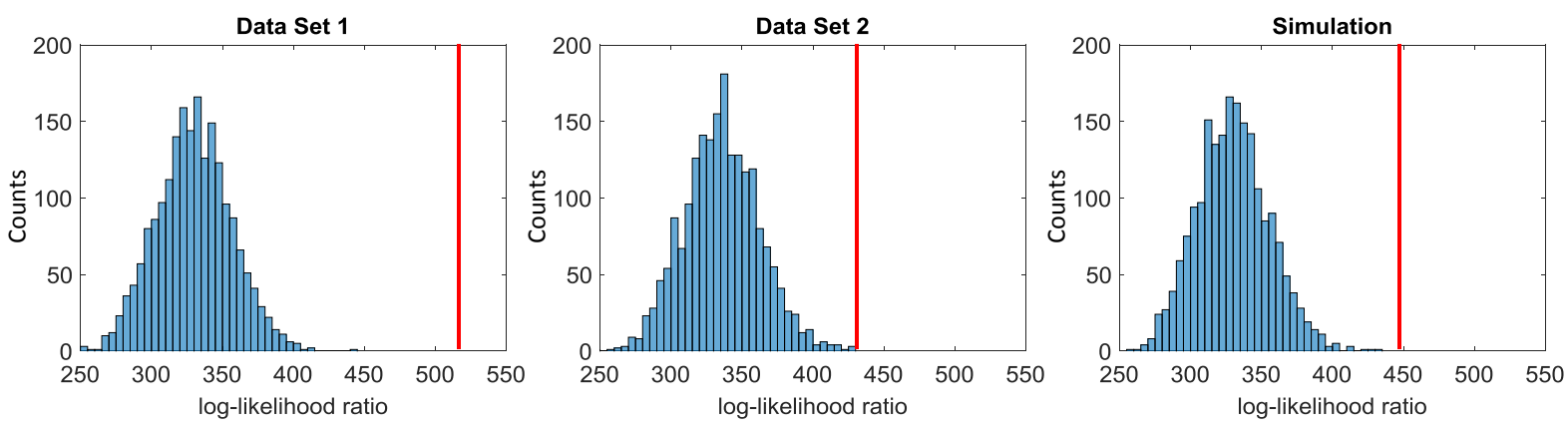

Figure S7: Likelihood ratio tests. Comparison of the log-likelihood ratio statistic $\lambda$ defined in Eq. 16 with the distribution of values of $\lambda$ obtained from 2000 parametric bootstrap resamples from the ML process. The deviation of the experimental value of $\lambda$ from the distribution are 7.1, 3.6, and 4.6 standard deviations for dataset 1 , dataset 2 , and a simulated data set, respectively. The simulated data set results from a simulation of the experiment that models systematic errors caused by drifts in the B single-qubit rotation angle, as explained in the text. 

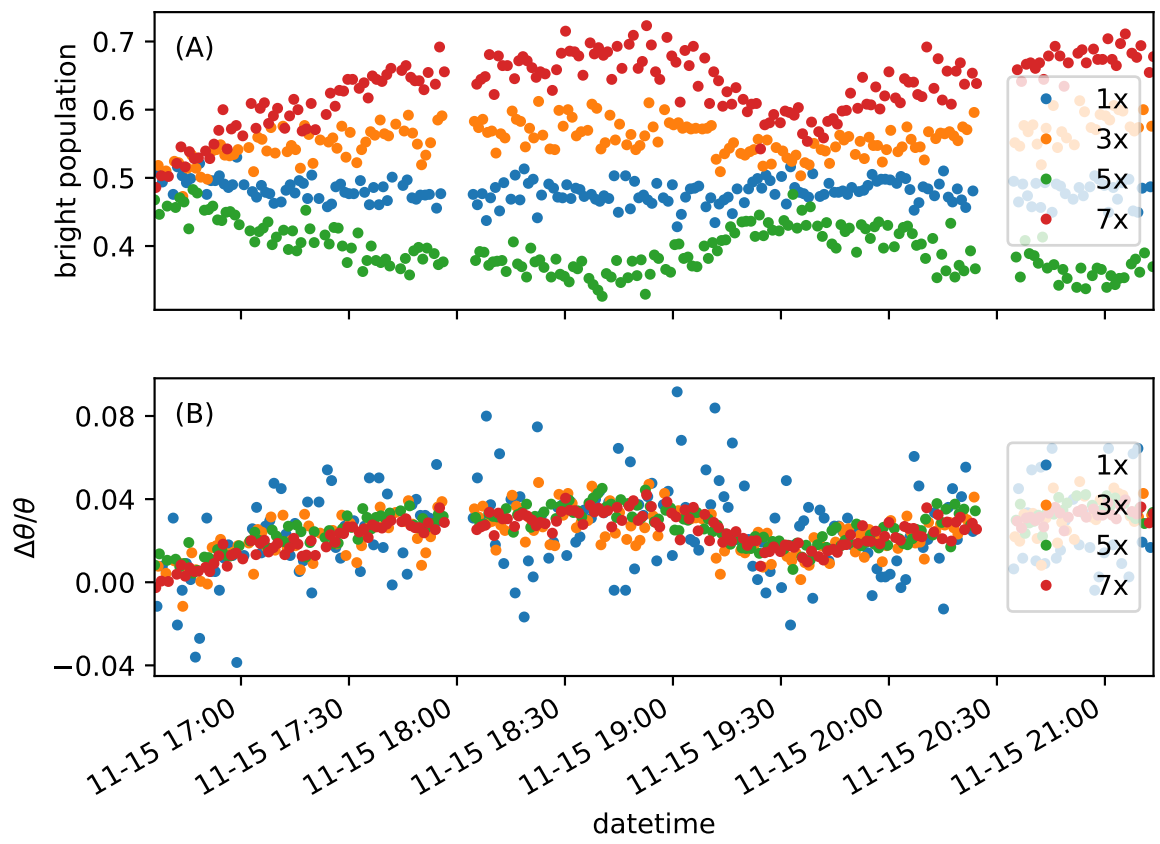

Figure S8: Drifts of single-qubit-rotation pulses. The rotation-angle drifts of single-qubitrotations on B ions are measured by applying 1 (blue), 3 (orange), 5 (green), and 7 (red) $\pi / 2$ pulses. The odd number of single-qubit-rotation pulses ideally excite the population to around 0.5 , a point where the slope of the population as a function of rotation angle is maximal, providing maximum sensitivity to rotation angle changes. Each single $\pi / 2$ pulse takes approximately $10 \mu \mathrm{s}$ with about $40 \mu \mathrm{s}$ gap between the pulses. Data taken on November 15, 2018. (A) Bright population after single-qubit rotation pulses. (B) Fractional changes of rotation angles derived from measured population. (See text) 

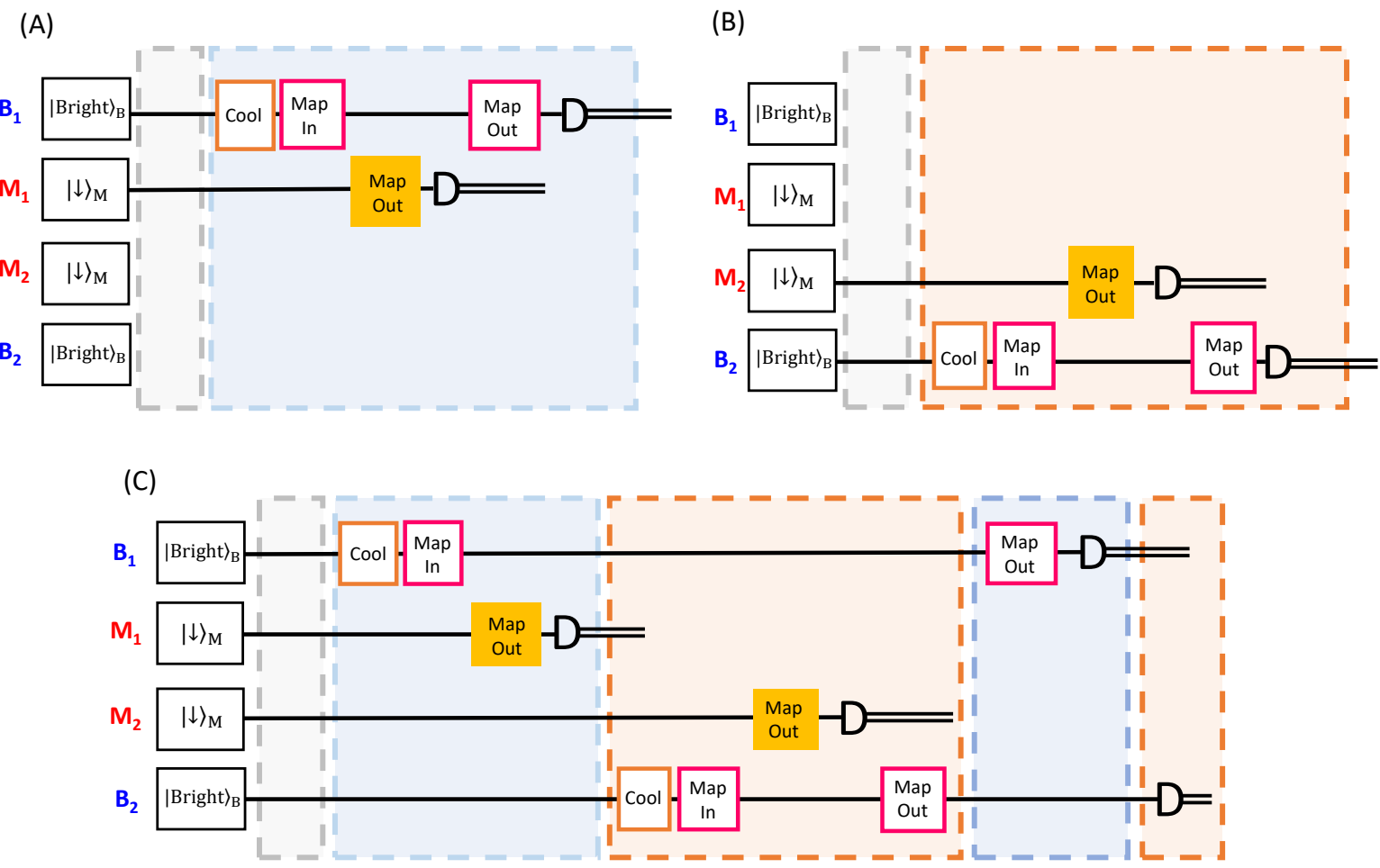

Figure S9: Experiments for determining SPAM and depumping errors. (A) and (B) Sequence for determining the SPAM errors for B and M ions detected in the double well potential. These are also the reference experiments for determining the stray-light-induced depumping errors. (C) To determine the stray-light-induced depumping error, cooling, state preparation, and measurement pulses are applied in the same order as in the QGT sequence to conduct SPAM measurements for all four qubits. These measurements contain the SPAM errors plus the straylight-induced depumping errors. The difference between this and the reference experiments in (A) and (B) gives the stray-light-induced depumping error. 
Table S1: Detailed steps of the QGT algorithm with the approximate durations of each step. The majority of the time is spent on cooling and shuttling the ions. DC: Doppler cooling, SP: state preparation, SBC: sideband cooling. The additional $\mathrm{M}$ ion detections in steps $\mathrm{E}$ and $\mathrm{F}$ are for diagnostics and are not part of the QGT algorithm.

\begin{tabular}{|c|c|c|c|}
\hline Step & Description & Operation & Duration \\
\hline \multirow{4}{*}{ A } & $\begin{array}{l}\text { Optical pumping and crystal initialization } \\
\text { of the four-ion chain }\end{array}$ & - & $3.2 \mathrm{~ms}$ \\
\hline & $\mathrm{DC}$ on $\mathrm{B}$ and $\mathrm{M}$ and $\mathrm{SP}$ of $\mathrm{M}$ ions & - & $1.3 \mathrm{~ms}$ \\
\hline & SBC on B ions & - & $5.3 \mathrm{~ms}$ \\
\hline & $\mathrm{M}_{1}-\mathrm{M}_{2}$ Bell-state generation & $\hat{F}$ & $220 \mu \mathrm{s}$ \\
\hline $\mathrm{B}$ & $\begin{array}{l}\text { Separation of four-ion chain into } B_{1}-M_{1} \\
\text { and } M_{2}-B_{2} \text { in double well }\end{array}$ & - & $570 \mu \mathrm{s}$ \\
\hline \multirow{4}{*}{$\mathrm{C}$} & Shifting double well & - & $230 \mu \mathrm{s}$ \\
\hline & $\mathrm{DC}, \mathrm{SBC}$, and $\mathrm{SP}$ of $\mathrm{B}_{1}$ & - & $2.2 \mathrm{~ms}$ \\
\hline & CNOT on $\mathrm{B}_{1}-\mathrm{M}_{1}$ & CNOT & $280 \mu \mathrm{s}$ \\
\hline & Map out and detection of $\mathrm{M}_{1}$ & - & $650 \mu \mathrm{s}$ \\
\hline \multirow{7}{*}{$\mathrm{D}$} & Shifting double well & - & $460 \mu \mathrm{s}$ \\
\hline & Cooling and $\mathrm{SP}$ of $\mathrm{B}_{2}$ & - & $2.2 \mathrm{~ms}$ \\
\hline & Conditional rotation on $\mathrm{M}_{2}$ & $\hat{R}(\pi, 0)$ & $25 \mu \mathrm{s}$ \\
\hline & CNOT on $\mathrm{B}_{2}-\mathrm{M}_{2}$ & CNOT & $280 \mu \mathrm{s}$ \\
\hline & Rotation on $\mathrm{M}_{2}$ & $\hat{R}(\pi / 2,-\pi / 2)$ & $15 \mu \mathrm{s}$ \\
\hline & Map out and detection of $\mathrm{M}_{2}$ & - & $650 \mu \mathrm{s}$ \\
\hline & Map out $\mathrm{B}_{2}$ & - & $220 \mu \mathrm{s}$ \\
\hline \multirow{5}{*}{$\mathrm{E}$} & Shifting double well & - & $460 \mu \mathrm{s}$ \\
\hline & $\mathrm{DC}$ on $\mathrm{M}_{1}$ & - & $200 \mu \mathrm{s}$ \\
\hline & Conditional phase shift on $\mathrm{B}_{1}$ & $\hat{R}_{\mathrm{Z}}(\pi)$ & $100 \mathrm{~ns}$ \\
\hline & Map out and detection of $\mathrm{B}_{1}$ & - & $540 \mu \mathrm{s}$ \\
\hline & Detection of $\mathrm{M}_{1}$ & & $180 \mu \mathrm{s}$ \\
\hline \multirow{5}{*}{$\mathrm{F}$} & Shifting double well & - & $460 \mu \mathrm{s}$ \\
\hline & $\mathrm{DC}$ on $\mathrm{M}_{2}$ & - & $200 \mu \mathrm{s}$ \\
\hline & Detection of $\mathrm{B}_{2}$ & - & $300 \mu \mathrm{s}$ \\
\hline & Detection of $\mathrm{M}_{2}$ & - & $180 \mu \mathrm{s}$ \\
\hline & Recombination & - & $800 \mu \mathrm{s}$ \\
\hline
\end{tabular}


Table S2: Entanglement fidelities and confidence intervals. Entanglement fidelities and confidence intervals of the teleported CNOT gate are determined using maximum likelihood (ML) estimation and a linear estimator.

\begin{tabular}{lcc} 
& dataset 1 & dataset 2 \\
\hline Fidelity of ML process & 0.858 & 0.851 \\
Fidelity (68\% CI from ML) & {$[0.858,0.871]$} & {$[0.852,0.866]$} \\
Fidelity (95\% CI from ML) & {$[0.852,0.878]$} & {$[0.845,0.872]$} \\
Fidelity from linear estimator $\hat{F}_{L}$ & 0.871 & 0.866 \\
Fidelity (68\% CI from linear estimator) & {$[0.860,0.882]$} & {$[0.856,0.878]$} \\
Fidelity (95\% CI from linear estimator) & {$[0.851,0.892]$} & {$[0.845,0.888]$}
\end{tabular}

Table S3: SPAM errors measurements. The uncertainties are determined from the standard deviation of multiple measurements.

\begin{tabular}{ccccc} 
& $\epsilon_{X, \uparrow}\left(10^{-2}\right)$ & $\epsilon_{X, \downarrow}\left(10^{-2}\right)$ & $\bar{\epsilon}_{X}\left(10^{-2}\right)$ & $\epsilon_{X, \text { Bell }}\left(10^{-2}\right)$ \\
\hline $\mathrm{B}_{1}$ & $0.5(4)$ & $0.4(2)$ & $0.5(2)$ & $0.7(3)$ \\
$\mathrm{B}_{2}$ & $1(1)$ & $0.4(2)$ & $0.7(6)$ & $1.0(9)$ \\
$\mathrm{M}_{1}$ & $0.2(1)$ & $1.4(4)$ & $0.8(2)$ & $1.2(3)$ \\
$\mathrm{M}_{2}$ & $0.2(1)$ & $1.3(3)$ & $0.7(2)$ & $1.1(3)$ \\
Both M ions (microwave) & $0.2(1)$ & $2.2(4)$ & $1.2(2)$ & $1.8(3)$
\end{tabular}

